\title{
ASPECTS OF CATEGORICAL RECURSION THEORY
}

\author{
PIETER HOFSTRA AND PHILIP SCOTT
}

\begin{abstract}
We present a survey of some developments in the general area of category-theoretic approaches to the theory of computation, with a focus on topics and ideas particularly close to the interests of Jim Lambek.
\end{abstract}

\section{Contents}

1. Introduction

1.1. On Lambek's Questions

1.2. General Notation and Background

2. What is a computable function?

2.1. The primitive recursive functions

2.2. The computable functions

2.3. Some Newer Models of Computability

3. Lambek's Categorical Proof Theory

3.1. A brief history

3.2. Internal Languages and free categories

3.3. CCCs and the Curry-Howard-Lambek correspondence

3.4. Elementary toposes and HAH

4. What are computable functions in categories?

4.1. Natural Numbers Objects and Prim

4.2. Representability

4.3. Going beyond the primitive recursive functions: free CCCs

4.4. Some properties of the free topos 16

4.5. C-monoids and Untyped Lambda Calculi 17

4.6. Plotkin's characterization of Kleene's $\mu$-recursion 18

5. Abstract Computability 19

5.1. Categories of Partial Maps $\quad 19$

5.2. Turing Categories 20

5.3. Computable maps and PCAs 21

6. Realizability 23

6.1. Kleene Realizability 23

6.2. Realizability Toposes 24

6.3. PCAs and Toposes 25

$\begin{array}{ll}\text { 7. Other Directions } & 26\end{array}$

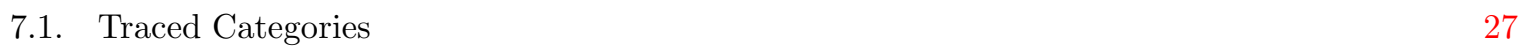

7.2. Typed PCAs 27

7.3. Computation at higher types 28

7.4. Higher-order computation in toposes 29

7.5. Complexity Theory 30

Conclusion $\quad 31$

$\begin{array}{ll}\text { References } & 31\end{array}$

Both authors are partially supported by an NSERC Discovery Grant. 


\section{INTRODUCTION}

Algorithms have been discussed for thousands of years, starting with the Babylonians and later the Greeks (e.g. Plato's academy, Euclid in Alexandria, etc.). These ideas were subsequently passed to (or rediscovered in) many mathematical cultures and civilizations (see [3]). Indeed, the word algorithm itself comes from the Latinized name of the author of a book on Hindu arithmetic, the Persian mathematician Muhammed ibn-Mūsā al-Khwārizmī (c. 825). Yet it was only in the 19th century that serious approaches to understanding the foundations of algorithms and computable functions began. For example, the modern idea of defining functions by iteration and proofs by induction seems to have originated in the writings of Richard Dedekind [28]. David Hilbert's seminal lectures on the foundations of mathematics led Thoralf Skolem in the early 1920's to axiomatize the primitive recursive functions, a class of inductively defined numerical functions which were intuitively computable. Was this all of them? Alas, no: a routine application of Cantor's diagonal argument ([27], p.91) shows that there are intuitively computable functions which are not primitive recursive. Indeed, in 1928 Hilbert's student Wilhelm Ackermann constructed an explicit example of a recursively defined, intuitively computable function which grows faster than any primitive recursive function. Throughout the 1920's Hilbert discussed the Entscheidungsproblem (Decision Problem) for predicate logic, whose surprising final (negative) answer was obtained independently by Alonzo Church and Alan Turing in 1936, influenced by work of Kurt Gödel (1931). Indeed, this was the culmination of seminal research developing the modern theory of computability and computable functions by the logicians Church, Gödel, and Church's students Stephen Kleene, J. Barkley Rosser, and Alan Turing in the period 1931-1936.

Jim Lambek, in his writings and public presentations, had a long-time interest in the foundations of computability and its history [3]. His published papers include his well-known introduction of abacuses in 1961 [65] as a simple model of computation (an alternative to Turing machines) as well as his work on applying Gerhard Gentzen's cut-elimination algorithm and normal forms to categorical coherence theory $[67,69,70]$. This led to his interest in typed combinatory algebras, typed lambda calculi and categorical theories of computation (see [72] and Part III of the book with the second author [77]). In linguistics and anthropology, as well as in mathematics, he often expressed interest in doing computation via relation algebras, e.g. from kinship terminology [8] to Mal'cev categories [15] to exact completions and partial equivalence relations [75]. We shall further explore some of these ideas in Section 3 below.

Here we shall examine three particular questions that occupied Lambek for many years.

(1) Are there natural recursion theories?

(2) What are the computable functions and functionals in various concrete categorical structures?

(3) Are there intrinsic algebraic/categorical approaches to recursion theory?

The detailed discussion of these questions will be pursued in the following sections. As a warm up, we describe the informal meaning of Lambek's questions (and, in part, some of the associated answers).

1.1. On Lambek's Questions. Concerning questions (1) and (2), in many conversations and lectures Lambek emphasized that "natural" recursion theories (and their classes of computable functions) should arise by examining the computable numerical functions in various free categories arising in categorical logic. Here, by a free structured category (where the kinds of structure we may wish to consider include monoidal structure, finite limits, cartesian closed structure, etc.) we mean the structured category with natural numbers object (NNO) freely generated by the empty graph. Such a category is then initial among the categories with this structure. We can define the notion of a representable numerical function analogously to how this is done in mathematical logic (as in Gödel [38], cf. also [77]) and we can ask: which numerical functions (partial functions, functionals, etc.) are representable in the various relevant free categories?

To this end, these questions were taken up in Lambek and Scott ([77], Part III) for categories associated to various higher-order logics, and will be discussed in more detail in Section 4 below. We summarize some of the early literature in Figure 1. 


\begin{tabular}{|c|c|}
\hline Free Categories & Definable Functions and Functionals \\
\hline \hline Cartesian and monoidal with NNO & Primitive recursive functions ([108, 100]) \\
\hline Cartesian closed with NNO & Gödel's Dialectica Functionals ([77]) \\
\hline The free elementary topos & $\begin{array}{c}\text { Provably total functions of HAH }([77]) \text { and } \\
\text { Higher provably recursive functionals ([109]) }\end{array}$ \\
\hline \hline C-monoids and CCCs \\
with reflexive objects & $\begin{array}{c}\text { Church's untyped lambda calculus (with surjective } \\
\text { pairing) and the partial recursive functions ([77]) }\end{array}$ \\
\hline
\end{tabular}

Figure 1. Natural Recursion Theories and their computable function(al)s

Concerning Question (3), since the 1960's there has been increasing interest in developing general categorical frameworks for computability theory. We mention here in particular the early work by Eilenberg and Elgot [32] on recursiveness, and the groundbreaking work by Di Paola and Heller on recursion categories [98, 45, 99], the modern incarnation of which we shall discuss in Section 5. Other aspects of computation and computability were studied from a categorical standpoint by various authors; for example, Lawvere [79] (see also [119]) gives a general version of the diagonal argument from which the well-known first recursion theorem, the fixed point theorem in untyped lambda calculus, and Gödel's diagonalization lemma can be obtained; while Mulry [96] introduces the recursive topos as a natural setting to consider a generalization of the Banach-Mazur functionals to all higher types.

We shall not attempt to give a full historical account of the large recent literature on categorical recursion theory. Instead, we shall focus on a few areas that the authors have become involved in which represent new directions of independent interest, but which also overlap with Lambek's interests.

1.2. General Notation and Background. We now introduce some notation and terminology for some of the structure appearing frequently in this paper. We assume that the reader is familiar with basic category theory. Standard references include [88, 5]. Some familiarity with lambda calculus [6] and the basic theory of computation [27, 97] is also an advantage.

The (large) category of sets and functions is denoted by Set. The category of sets and partial functions (that is, single-valued relations) is denoted by Par. By Rel, we mean the category of sets and relations.

An idempotent is an endomorphism $e: A \rightarrow A$ for which $e e=e$. An idempotent $e$ splits when there is an embedding-retraction pair $m: B \rightarrow A, r: A \rightarrow B$ with $r m=1_{B}, m r=e$. The idempotent splitting or Karoubi envelope of $\mathcal{C}$ is the category $\mathcal{K}(\mathcal{C})$ whose objects are the idempotents of $e$, and whose morphisms $(A, e) \rightarrow(B, d)$ are maps $f: A \rightarrow B$ with $d f=f=f e$. More generally, for $E$ a set of idempotents, $\mathcal{K}_{E}(\mathcal{C})$ is the full subcategory of $\mathcal{K}(\mathcal{C})$ on the objects determined by $E$. Finally, a retract of an object $A$ is an object $B$ together with an embedding-retraction pair $m: B \rightarrow A, r: A \rightarrow B$, with $r m=1_{B}$. We write $B \triangleleft A$ to indicate that $B$ is a retract of $A$.

\section{What is a COMPUtable FUnCtion?}

As explained in the introduction, one of the fundamental challenges for mathematical logic in the first quarter of the 20th century was to make precise the notions of computation, computable function, and computable set. ${ }^{1}$ This section reviews some of these developments of the classical theory, setting the stage for the categorical approaches to be introduced later.

\footnotetext{
${ }^{1}$ Originally, the terminology recursive function has been prevalent, due to the emphasis on the use of recursive procedures. Indeed, many authors have referred to the subject as recursion theory. However, as argued in [112], the term computability more aptly captures the flavour of the subject, and also emphasizes inclusion of other notions or models of computation.
} 
2.1. The primitive recursive functions. As summarized in the Introduction, historically the attempts to define computability focussed on iterative or recursive procedures. These seem to have been first analyzed in the writings of Dedekind in the 19th century [28]. A formal system of Primitive Recursive Arithmetic, concomitant with Hilbert's foundational lectures in the 1920's, was developed by Skolem [111]. Rózsa Péter's work in the early 1930s (later presented as [101]) is considered to have provided the foundations for the theory of recursive functions; many of the central ideas were further developed in detail by Hilbert-Bernays [47], and especially Goodstein [40]. Moreover, these functions were also used by Gödel in his famous Incompleteness Theorem paper [38].

Consider total numerical functions $\mathbb{N}^{k} \rightarrow \mathbb{N}, k \geq 1$. We recall the traditional definition of primitive recursion, then include a somewhat non-standard definition by Lambek.

Definition 2.1 (Primitive Recursive Functions). The primitive recursive functions are the smallest class Prim of numerical functions generated from Basic Functions by composition (or substitution) and primitive recursion.

The Basic Functions are the constant zero function $Z(x)=0$, the successor function $S(x)=x+1$, and the projection functions $U_{i}^{n}: \mathbb{N}^{n} \rightarrow \mathbb{N}, U_{i}^{n}\left(x_{1}, \ldots, x_{n}\right)=x_{i}$. The closure rules are as follows $(\vec{x}$ denotes an element of $\left.\mathbb{N}^{n}\right)$ :

- Composition: if $f_{1}: \mathbb{N}^{n} \rightarrow \mathbb{N} \in$ Prim and $g\left(u_{1}, \cdots, u_{k}\right): \mathbb{N}^{k} \rightarrow \mathbb{N} \in$ Prim, then $\operatorname{comp}\left(g, f_{1}, \ldots, f_{k}\right)$ : $\mathbb{N}^{n} \rightarrow \mathbb{N} \in$ Prim, where

$$
\operatorname{comp}\left(g, f_{1}, \ldots, f_{k}\right)(\vec{x})=g\left(f_{1}(\vec{x}), \ldots, f_{k}(\vec{x})\right) .
$$

- Primitive Recursion: if $g(\vec{x}), h(\vec{x}, y, u) \in \operatorname{Prim}$ then so is $\operatorname{rec}(\vec{x}, y)$, where

$$
\operatorname{rec}(\vec{x}, 0)=g(\vec{x}), \operatorname{rec}(\vec{x}, S(y))=h(\vec{x}, y, \operatorname{rec}(\vec{x}, y)) .
$$

Using this, we may also define a relation $R \subseteq \mathbb{N}^{k}$ to be primitive recursive when its characteristic function is. Most of the numerical functions and relations used in everyday mathematics are primitive recursive.

Let us mention a somewhat nonstandard definition ${ }^{2}$ of primitive recursive functions, introduced by Lambek in [3], p. 246. Following Lambek, we elide function arguments, writing e.g. $f \vec{x} u \vec{v}$ for $f(\vec{x}, u, \vec{v})$, etc.

Definition 2.2 (Lambek's Primitive Recursive Functions). (i) Basic functions: Identity Ix $=x$, Successor $S x=x+1$, and Zero $Z x=0$.

(ii) Generating Rules:

(a) Substitution: given functions $f \vec{x} u \vec{z}$ and $g \vec{y}$ we can form $h \vec{x} \vec{y} \vec{z}=f \vec{x}(g \vec{y}) \vec{z}$.

(b) Interchanging two arguments: given $f \vec{x} u v \vec{y}$, we can form $g \vec{x} u v \vec{y}=f \vec{x} v u \vec{y}$.

(c) Contracting two arguments: given $f \vec{x} u v \vec{y}$, we can form $g \vec{x} u \vec{y}=f \vec{x} u u \vec{y}$.

(d) Introducing dummy arguments: given $f \vec{x} \vec{y}$, we can form $g \vec{x} u \vec{y}=f \vec{x} \vec{y}$.

(e) Primitive Recursion: given $g \vec{x}$ and $h \vec{x} y z$, we can form $f \vec{x} y$, where $f \vec{x} 0=g \vec{x}, f \vec{x}(S y)=$ $h \vec{x} y(f \vec{x} y)$.

Note that Lambek's rules generating Prim are closely related to the Curry-Howard functional interpretation of intuitionistic sequent calculus proofs (with non-logical axioms). Indeed, consider a proof of an intuitionistic sequent $A_{1}, \cdots, A_{n} \vdash B$. The functional interpretation interprets the proof by functional "proof terms" (see Girard [37] and Lambek [73]) of the form

$$
x_{1}: A_{1}, \cdots, x_{n}: A_{n} \vdash f \vec{x}: B .
$$

The identity function interprets the identity sequent $A \vdash A$. The generating rules (a)-(d) above correspond respectively to interpreting the following rules of sequent calculus (by associating to proof terms

\footnotetext{
${ }^{2}$ We modify slightly the Basic functions, which were missing one.
} 
for each of the premises a proof term of the conclusion): cut (a), interchange (b), contraction (c) and weakening $(d)$.

$$
\begin{array}{ll}
\frac{\Gamma, A, \Delta \vdash C \quad \Sigma \vdash A}{\Gamma, \Sigma, \Delta \vdash C} \text { cut } & \frac{\Gamma, A, B, \Delta \vdash C}{\Gamma, B, A, \Delta \vdash C} \text { interchange } \\
\frac{\Gamma, A, A, \Delta \vdash B}{\Gamma, A, \Delta \vdash B} \text { contraction } & \frac{\Gamma, \Delta \vdash B}{\Gamma, A, \Delta \vdash B} \text { weakening }
\end{array}
$$

Finally, the zero function, successor, and primitive recursion (e) above may be thought-of as non-logical axioms or rules specifying a weak natural numbers object, i.e. a particular type $\mathrm{N}$. In this sense, we have distinguished proofs $\vdash 0: \mathrm{N}$ and $x: \mathrm{N} \vdash S x: \mathrm{N}$, and primitive recursion is a special case of the iterator (see Subsection 4.1 below).

2.2. The computable functions. So, does Computable $=$ Primitive Recursive? Alas, no, by a standard application of Cantor's diagonal argument. Indeed, the previously mentioned Ackermann function (which is computable but not primitive recursive) can be defined by a so-called double recursion scheme (see e.g. R. Péter's book [101]). So what is a computable function? This was taken up in a remarkable development in the years 1931-1937 (primarily centered around Princeton University) which, as it turned out, led to the foundations of modern computer science. Let us briefly recall the history.

- A. Church (1932-34) and his students (S. C. Kleene, J. B. Rosser) developed (untyped) lambda calculus as a model of computation (and, as later realized in the 1960's, a foundation of modern programming language theory). Church formulated Church's Thesis (1936): the intuitively computable numerical functions are exactly those you can compute in $\lambda$-calculus. This thus answered the age-old question we began with.

Originally, however, Church's thesis was not believed by Gödel (there being insufficient evidence at the time). However, in rapid developments, new evidence arose:

- Kleene (1934-35) developed the partial $\mu$-recursive functions: we add to Prim the following generating scheme on partial functions, called minimalisation: given $g(\vec{x}, y)$, we can form

$$
f(\vec{x})=\mu y \cdot g(\vec{x}, y)=0
$$

where $\mu y . g(\vec{x}, y)=0$ means the least $y$ such that $g(\vec{x}, y)=0{ }^{3}$ (If we wish to restrict to total functions, we add the proviso $\forall \vec{x} \exists y . g(\vec{x}, y)=0$.)

- Gödel-Herbrand (1934). Gödel lectured on an equation calculus to define "computable" functions, based in part on a letter from Herbrand. This is described in Kleene's book [61].

- Turing (1936) independently introduced Turing machines: an abstract mechanical computing device. He gave a convincing analysis of the meaning of being "computable" without restrictions on space or time. This led to Turing's thesis: the intuitively computable functions were those computable by Turing's abstract machines. This ground-breaking paper also showed the recursive unsolvability of Hilbert's Entscheidungsproblem, simultaneously and independently solved by Church in 1936 (who was inspired by his studies in untyped lambda calculus).

- Turing then became a student of Church at Princeton. During the period 1936-37, Church, Kleene and Turing carefully proved the "equivalence" of the above different models of computability, in the sense that all notions gave exactly the same class of computable functions! This work convinced Gödel of the truth of the Church-Turing thesis (CT). ${ }^{4}$

2.3. Some Newer Models of Computability. After the exciting results in the late 1930's, mathematicians continued the analysis of abstract theories of computing. For example Emil Post (1943) and Andrei Markov (1951) developed theories of computability based on string rewriting grammars (following in the footsteps of the Norwegian mathematician Axel Thue). These notions of computability turned out to be Turing complete, i.e., equivalent to Turing computability. In 1944, Post [105] also

\footnotetext{
${ }^{3}$ Provided for all $z<y, g(\vec{x}, z)$ is defined and not $=0$. If there is no such $y, \mu y \cdot g(\vec{x}, y)=0$ is undefined.

${ }^{4} \mathrm{CT}$ is not a mathematical statement: it is an experimental statement, identifying an informal class (namely, the "intuitively computable" numerical functions) with a precise mathematical class of functions.
} 
initiated the systematic study of the recursively enumerable sets (previously defined by Kleene and Church in terms of images of recursive functions), in particular the study of the r.e. degrees.

A particularly interesting period in the more recent modelling arose in 1960-61 (simultaneously and almost independently): the development of Unlimited Register Machines. Within a period of a few months, papers by J. Lambek, Z. Melzak, M. Minsky, and (slightly delayed) J. Shepherdson and J. Sturgis introduced this influential model of computability. ${ }^{5}$

Lambek's paper [65] was by far the simplest to read of all the papers on Register Machines, and used a highly graphical syntax, akin to flowcharts. Register machines were particularly influential pedagogically, compared to the intricacies of Turing machines. A direct translation between Lambek's machine models and Turing Machines is given in Boolos and Jeffrey [11].

Let us briefly recall the formalism. A Lambek abacus consists of a series of Locations (or registers) of arbitrary capacity (denoted $X, Y, Z, \cdots$ ), into which we may put (or remove) pebbles, called Counters. We assume an unlimited supply of (indistinguishable) pebbles as counters. There are a small number of Elementary Instructions for building abacuses, as follows:

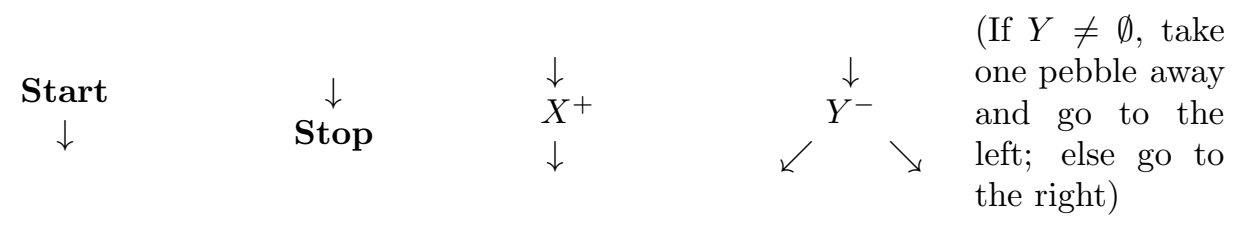

Figure 2. Abacus instructions

Here $X^{+}$denotes the operation of adding one pebble to location $X$. Programs are formed from a finite number of instructions, arranged in a flow chart (directed graph) with root Start, possibly with feedback loops. In section 7.1 we will discuss the categorical semantics of such a graphical notion of computation.

\section{Lambek's Categorical Proof Theory}

Categorical logic is concerned with the study of classes of categories with additional categorical structure, such as categories with finite limits, regular categories, monoidal (closed) categories, cartesian closed categories, first-order categories, toposes, and so on. Ideally, such a class of categories corresponds to a well-behaved fragment of logic; for example, cartesian closed categories correspond to typed lambda calculus (see below). This correspondence means that there is a sound and complete interpretation of the logic in this class of categories. On the one hand, this allows us to use proof-theoretic techniques (rewriting for example) to reason about categorical structure, while on the other hand we may apply categorical results to obtain information about logical systems. Categorical proof theory is particularly concerned with the study of syntactically generated categories and their properties. This section describes some of the contributions due to Lambek, as well as some related developments.

3.1. A brief history. Lambek's early works in mathematical linguistics [64, 66] as well as his later work in categorical coherence theory [67, 69, 70] employed proof theory, notably Gentzen's sequent calculi. Coherence theorems in category theory were aimed at answering the following very general question: (see Mac Lane [88]) given a freely generated structured category $\mathcal{C}$, prove that every diagram (built from some canonical morphisms) commutes. Lambek reformulated the question more generally as follows:

(i) Given a freely generated structured category $\mathcal{C}$, how do we effectively generate the hom-sets $\operatorname{Hom}_{\mathcal{C}}(A, B) ?$

\footnotetext{
${ }^{5}$ Lambek's and Melzak's papers appeared back to back in the same issue of the Bulletin of the Canadian Mathematical Society. Lambek's paper is a considerable simplification of Melzak's approach.
} 
(ii) Find an effective method to solve the word problem for hom-sets in such $\mathcal{C}$. In particular, any two morphisms with the same domain and codomain generated from the canonical morphisms must be equal.

Lambek's seminal idea was to reformulate this problem using proof-theory, then apply Gentzen's Cut-Elimination (or Normalization) theorems. Namely, he considered freely generated monoidal or residuated categories as kinds of "logics" or "labelled deductive systems": the objects of such categories are "formulas" (freely generated from some atomic ones), while arrows would then be equivalence classes of proofs (or proof trees).

In particular, an arrow $f \in \operatorname{Hom}_{\mathcal{C}}(A, B)$ would be considered as a proof of the Gentzen sequent $A \vdash B$, while composition of arrows $f: A \rightarrow B$ and

$g: B \rightarrow C$ to obtain $g \circ f: A \rightarrow C$ becomes an instance of the Cut-Rule. The equations of a category force one to impose the notion of "equality of proofs". Algebraically, one generates a congruence relation on proofs (or better, between proof trees).

For (i), we generate all proofs of the sequents $A \vdash B$, by Gentzen's proof search. For the word problem (ii), Gentzen's cut-elimination methods amount to introducing a compatible rewriting system on proofs. To decide if two proof trees denote the same arrow or not, reduce each to a unique normal (or cut-free) form. The problem of deciding equality of arrows amounts to deciding if their normal forms are identical or not. ${ }^{6}$

Lambek pursued these ideas in the late 1960's and early 1970's using cut-elimination to solve the word problem for (among others) residuated and biclosed monoidal categories in [67, 69, 70]. But it was soon realized by proof theorists, beginning with G. Mints [93], that natural deduction calculi (and their associated lambda calculi of proof-terms, under normalization) leads to a smoother technical framework for such word problems. Mints and his students greatly increased the scope of Lambek's proof-theoretic approaches to coherence, influencing even Kelly and Mac Lane [59]. Normalization approaches to coherence/decision problems for monoidal categories (using reduction of lambda-like proof terms) were first investigated by Mints and his students ([93, 94], reprinted in [95]). In the case of monoidal closed categories, it was shown in Mac Lane [87] that Mints' proof-theoretic methods agreed almost exactly with the approach to coherence due to Kelly and Mac Lane, all of which in turn were influenced by Lambek's original use of Cut-Elimination.

Meanwhile, in the 70s and 80s, Lambek's own algebraic studies on functional completeness and combinatory logics [71, 72], led him to consider connections of lambda calculi to freely generated cartesian and cartesian closed categories. Around the same time, work in computer science in applying lambda calculi and natural deduction to functional languages led to the now-common practice of assigning lambda- (or proof-) terms to proof trees [37]. Hence "equality of proofs" becomes provable equality of the associated terms assigned to the proof trees. This is sometimes known as the Curry-Howard-Lambek correspondence, to be discussed in more detail below.

After the introduction of Girard's Linear Logic in 1986 [36] (which used sequent calculi and gives particular analysis of the structural rules) Lambek realized his earlier work in linguistics amounted to a kind of substructural (linear) logic without structural rules. He introduced generalizations of deductive systems to more general Gentzen sequents with their associated multicategories and term calculi [73]. On the subject of categorical proof theory, cut-elimination and applications to (structured) monoidal categories, linear logics, coherence theorems, et cetera, there has been an explosion of activity. As a small sample of the extensive literature, we mention works of R. Blute, R. Cockett, R. Seely and co-workers [10, 26, 9] K. Došen, et al. [29, 30, 31], B. Jay [54, 55].

3.2. Internal Languages and free categories. As mentioned above, coherence problems are often formulated in terms of free categories. Let us make this more precise. Suppose that S-Cat is a category whose objects are structured categories and whose morphisms are structure-preserving functors. There

\footnotetext{
${ }^{6}$ An equivalent formulation [114] of a coherence theorem for a free category of some kind says: given any two objects $A, B$, there is at most one proof (built from canonical arrows) of the associated sequent $A \vdash B$.
} 
is a forgetful functor

\section{S-Cat $\longrightarrow$ DirGrph}

to the category of directed graphs. The free structured category generated by a (small) graph $\mathbb{G}$, denoted $\mathcal{F}(\mathbb{G})$, can be described in terms of a left adjoint to this forgetful functor. In [77] this left adjoint is constructed using logical syntax along the following lines.

(i) One sets up an equivalence of categories S-Cat $\stackrel{\simeq}{\longrightarrow}$ Lang where Lang is some category of formal theories (whose morphisms are "interpretations" which preserve the structure exactly). The equivalence is implemented by a pair of functors: $L: \mathbf{S - C a t} \longrightarrow$ Lang which associates to every category $\mathcal{C}$ a so-called internal language and $C:$ Lang $\longrightarrow$ S-Cat, which associates to a language $\mathcal{L}$, a category $C(\mathcal{L})$, called the (syntactic) category generated by $\mathcal{L}$.

(ii) Next, one constructs, given a directed graph $\mathbb{G}$, the theory $\mathcal{L}_{\mathbb{G}}$ generated by $\mathbb{G}$. The types of $\mathcal{L}_{\mathbb{G}}$ are generated from the nodes of $\mathbb{G}$, while the terms are generated using the term-formation rules of the logic by including the arrows of $\mathbb{G}$ as term-forming operations. The free structured category $\mathcal{F}(\mathbb{G})$ generated by $\mathbb{G}$ may then be taken to be $C\left(\mathcal{L}_{\mathbb{G}}\right)$, the syntactic category of $\mathcal{L}_{\mathbb{G}}$. We thus have the following picture:

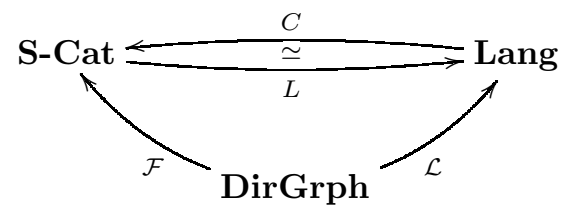

Of particular importance is the case where $\mathbb{G}$ is the empty graph. The resulting category $\mathcal{F}(\mathbb{G})$ is then the initial structured category.

In the book [77], such theories include typed (and even untyped) lambda calculi (corresponding to cartesian closed categories with additional structure) and intuitionistic higher order logics (Russellian type theories) with full impredicative comprehension, extensionality, and Peano's axioms (corresponding to elementary toposes with logical morphisms and natural numbers). We briefly discuss the two cases of Cartesian Closed Categories and Elementary Toposes (both with natural numbers object) below.

It is important to note that in order to obtain a 1-categorical equivalence $\mathbf{S}-\mathbf{C a t} \simeq \mathbf{L a n g}$ of this kind, we need to consider the objects of S-Cat not just as structured categories, but as categories equipped with specified structure. Similarly, we require the functors to preserve this specified structure on the nose. It is possible to avoid working with chosen structure, but then one should instead consider $\mathbf{S}-\mathbf{C a t}$ as a 2-category, and set up a 2-categorical equivalence with a suitable 2-category of theories. An example of this finer analysis appears (in this volume) in the paper of Castellan et al. [17], which discusses the Seely correspondence between locally cartesian closed categories and dependent type theories, and, more generally, provides a suitable 2-categorical perspective on categorical logic.

3.3. CCCs and the Curry-Howard-Lambek correspondence. Cartesian closed categories were introduced by Lawvere in the early 1960s as the categorical analog of Church's typed lambda calculi. In the early 1970s, Lambek explored this correspondence, along with connections to Schönfinkel and Curry's works on combinatory algebras and functional completeness. The precise tripartite categorical equivalence of cartesian closed categories, typed lambda calculi, and labelled deductive systems for positive intuitionistic propositional calculus (modulo equality of proofs) was developed in detail in [77]. This yields a modern version of the so-called Curry-Howard correspondence [37], with the additional idea (Lambek [67, 69]) of equations between proofs, and is summarized in Theorem 3.7 below.

Definition 3.1. A cartesian closed category $\mathcal{C}$ (with specified structure) is a cartesian category $\mathcal{C}$ (i.e., a category with specified finite products) such that, for each object $A \in \mathcal{C}$, the functor $(-) \times A: \mathcal{C} \rightarrow \mathcal{C}$ has a specified right adjoint, denoted $(-)^{A}$. Thus, there is a natural isomorphism (natural in $B$ and $C$ ):

$$
\operatorname{Hom}_{\mathcal{C}}(C \times A, B) \cong \operatorname{Hom}_{\mathcal{C}}\left(C, B^{A}\right) .
$$


Example 3.2. Examples of CCCs The category of sets is a CCC with $B^{A}$ the set of all functions $A \rightarrow B$. More generally, any functor category $\left[\mathcal{C}^{\text {op }}\right.$, Set $]$ is a CCC, where $G^{F}(C)$ is the set of natural transformations from $\operatorname{Hom}_{\mathcal{C}}(-, C) \times F$ to $G$. The category Cat of small categories is also cartesian closed, as are many categories of "nice" topological spaces, such as compactly generated Hausdorff spaces.

Next, consider simply typed lambda calculi.

Definition 3.3. A simply typed lambda calculus consists of the following data. First, it has a collection of simple types generated from a set of ground types $G$ by the grammar

$$
\text { Types } \quad A, B::=G|1| A \times B \mid A \Rightarrow B \text {. }
$$

At each type, we assume given an infinite set of variables; we write $x: A$ to indicate that $x$ is a variable of type $A$. Next, we have, for all types $A_{1}, \ldots, A_{k}, B$ a (possibly empty) set of basic terms $E\left(A_{1}, \ldots, A_{k} ; B\right)$. Then the collection of typed terms is generated using the rules displayed in Figure 3. We make the usual assumptions (see e.g. $[6,77]$ ) regarding free and bound variables, and write $F V(t)$ for the set of free variables of $t$; each $x \in F V(t)$ has a unique type, and from the term $t$ we can recover the types of the free variables in $t$.

$$
\begin{array}{ccc}
\overline{*: 1} & \frac{f \in E\left(A_{1}, \ldots, A_{k} ; B\right) x_{i}: A_{i}}{f\left(x_{1}, \ldots, x_{k}\right): B} & \frac{x: A \quad \varphi(x): B}{\lambda x: A \cdot \varphi(x): B^{A}} \\
\frac{a: A \quad b: B}{\langle a, b\rangle: A \times B} & \frac{t: A_{1} \times A_{2}}{\pi_{i} t: A_{i}} & \frac{f: B^{A} t: A}{f t: B}
\end{array}
$$

Figure 3. Lambda Calculus Terms

Finally, we have equations between terms of the same type. We write $t={ }_{X} s: A$ to express that the terms $t, s$ are equal, and that the free variables of $t$ and $s$ are contained in the set $X$.

The relations $=_{X}$ are congruences satisfying the following clauses ${ }^{7}$ :

- $t=_{X} s, X \subseteq Y$ implies $t==_{Y} s$

- $t={ }_{X} s$ implies $f t={ }_{X} f s$ (where $f: B^{A}$ and $t, s: B$ )

- $\varphi(x)={ }_{X \cup\{x\}} \psi(x)$ implies $\lambda x . \varphi(x)={ }_{X} \lambda x . \psi(x)$

- $a==_{X} *($ where $a: 1)$

- $\pi_{i}\left\langle a_{1}, a_{2}\right\rangle={ }_{X} a_{i} ; \quad a={ }_{X}\left\langle\pi_{1} a, \pi_{2} a\right\rangle$

- $(\beta) \quad(\lambda x . \varphi(x)) t==_{X} \varphi[t / x] ; \quad(\eta) \quad f=_{X} \lambda x . f x$ where $x \notin F V(f)$

It is possible to augment simply typed lambda calculus with additional types, terms, and equations (cf. [77]). We discuss the case of adding natural numbers and lists in Subsection 4.1 below.

An important example of a simply typed lambda calculus arises as follows.

Definition 3.4 (Simply typed $\lambda$-calculus from a graph). Consider a directed graph $\mathbb{G}=(G, E)$. The calculus $\mathcal{L}_{\mathbb{G}}$ has as ground types the vertices of $\mathbb{G}$, and as basic terms the edges of $\mathbb{G}$, (i.e. whenever $f: A \rightarrow B$ is in $E$, there is a basic term $f(x): B$, with $x: A$.) The congruence $t={ }_{X} s$ on terms is the smallest congruence satisfying the rules of simply typed lambda calculus.

\footnotetext{
${ }^{7}$ Here we present lambda calculi as ordinary equational theories, as in [77]. One could also write equational logics in an appropriate sequent calculus, writing $t={ }_{X} s: A$ as $\vdash_{X} s=t: A$ (cf. Barendregt's lambda theories [6] and the use of HOL below).
} 
We now define the category CCC whose objects are CCCs (with chosen products and exponentials), and whose morphisms are functors preserving the chosen products and exponentials on the nose. On the other hand, we define the category Typed $\lambda$-calc to have typed lambda calculi as objects, and translations as morphisms. Here, a translation between two calculi is a mapping sending types to types and terms to terms, in such a way that all type and term formation operations are preserved and that provable equality between terms is preserved.

Definition 3.5 (Internal language of a $\mathrm{CCC}$ ). Let $\mathcal{C}$ be a cartesian closed category. The internal language of $\mathcal{C}$ is the simply typed lambda calculus $L(\mathcal{C})$ generated by the underlying graph of $\mathcal{C}$, together with all equations holding between arrows of $\mathcal{C}$.

In the other direction, we construct a $\operatorname{CCC} C(\mathcal{L})$ from a typed lambda calculus $\mathcal{L}$ :

Definition 3.6 (Syntactic Category). Let $\mathcal{L}$ be a simply typed lambda calculus. Define a category $C(\mathcal{L})$ by:

Objects: The types of $\mathcal{L}$.

Morphisms: For any term $t: T$ with $F V(t)=\left\{x_{1}: T_{1}, \ldots, x_{n}: T_{n}\right\}$, we have a morphism $[t]: T_{1} \times \cdots \times T_{n} \rightarrow T$. Here $[t]$ is the equivalence class of $t$ under provable equality of the theory $\mathcal{L}$.

Identities: The identity at an object $T$ is represented by the term $x: T$.

Composition: Given terms $t(x), s(y)$ representing morphisms $A \rightarrow B$ and $B \rightarrow C$ respectively (where we assume that $t$ is substitutable for $y$ in $s$ ), the term $s[t / y]$ (the result of substituting $t(x)$ for all variables $y$ in $s$ ) represents the composite $A \rightarrow C$.

We now have the promised result ${ }^{8}$ :

Theorem 3.7 (Curry-Howard-Lambek correspondence [77]). The pair of functors $L: \mathbf{C C C} \rightarrow \mathbf{T y p e d ~} \lambda$-calc (internal language) and $C:$ Typed $\lambda$-calc $\rightarrow \mathbf{C C C}$ constitute an equivalence of categories.

The above theorem extends to include adding the natural numbers and similar data types (of which the categorical aspects are discussed in the next Section).

3.4. Elementary toposes and HAH. We now outline another instance of an equivalence between a class of categories and a fragment of logic, namely elementary toposes with NNO and higher-order intuitionistic arithmetic $(\mathrm{HAH})$.

Recall that in a category $\mathcal{C}$, a subobject of an object $A$ is an equivalence class of monomorphisms $m: X \rightarrow A$, where two monomorphisms are equivalent precisely when they factor through each other. The collection of subobjects of $A$ is denoted $S u b(A)$. The assignment $A \mapsto S u b(A)$ is a contravariant functor from $\mathcal{C}$ to the category of posets.

A category is said to have canonical subobjects when every subobject has a chosen representative. In Set, for example, we may represent a subobject through its set-theoretic image.

Definition 3.8 (Elementary Topos). A category $\mathcal{C}$ is a topos when it has the following structure:

- $\mathcal{C}$ has finite limits

- $\mathcal{C}$ has power-objects: for each $A$ there exists an object $\mathrm{P} A$ and natural bijection

$$
\operatorname{Hom}_{\mathcal{C}}(B, \mathrm{P} A) \cong S u b(B \times A) .
$$

A power-object $\mathrm{P} A$ (when it exists) represents the functor $S u b(-\times A)$. In the category of sets, we may take $\mathrm{P} A$ to be the powerset of $A$, and then the defining bijection becomes the familiar correspondence between relations $R \subseteq B \times A$ and functions $r: B \rightarrow \mathrm{P} A$.

In a topos, we write $\Omega$ for $\mathrm{P} 1$. This is the subobject classifier: there is a natural bijection

$$
\operatorname{Hom}_{\mathcal{C}}(B, \Omega) \cong S u b(B) .
$$

\footnotetext{
${ }^{8}$ Lambek reported that when he lectured at Columbia announcing these results Sammy Eilenberg is reported to have said: "This is wonderful. Now category theorists will never have to learn lambda calculus!"
} 
We think of $\Omega$ as the object of truth values of $\mathcal{C}$, and of $\mathrm{P} A$ as the exponential $\Omega^{A}$.

The category of sets is of course a typical example of a topos, as are functor categories $\left[\mathcal{C}^{\text {op }}\right.$, Set $]$. Other examples will be discussed below. The qualifier elementary is used to stress the inclusion of toposes other than Grothendieck Toposes (which are required to be cocomplete and have a small set of generators). ${ }^{9}$

In the context of elementary toposes, one often considers logical morphisms between toposes. These are functors preserving all the topos structure. Just as for CCCs, we work with toposes with specified structure and morphisms strictly preserving this structure.

Definition 3.9. The category Top has:

Objects: Elementary toposes with specified finite limits and power objects, and with canonical subobjects.

Morphisms: Logical functors preserving all specified structure on the nose.

Next, let us describe (intuitionistic) higher-order logic (HOL). This formal system can be thought of as an extension of simply typed lambda calculus, with added type and term constructors for the type $\Omega$ of propositions and for power objects $\mathrm{P} A$. (However, we do not include exponentials explicitly, as they are definable in terms of the other operations ${ }^{10}$.) Thus the types are generated from ground types $G$ using the following grammar:

$$
\text { Types } \quad A, B::=G|1| A \times B|\Omega| \mathrm{P} A .
$$

The terms are generated from basic terms and variables using the rules displayed in Figure 4 (where we omit the rules already stated for simply typed lambda calculus in Figure 3):

\begin{tabular}{|ccc|}
\hline$\frac{a: T a^{\prime}: T}{a=a^{\prime}: \Omega}$ & $\frac{a: T \quad \alpha: \mathrm{PT}}{a \in \alpha: \Omega}$ & $\frac{x: A \quad \varphi(x): \Omega}{\{x: A \mid \varphi(x)\}: \mathrm{P} A}$ \\
\hline
\end{tabular}

Figure 4. Terms of higher-order intuitionist logic

In [77] there are two axiomatizations of higher order logic, including the one above based on equality (between terms of the same type), comprehension, extensionality, and (in case we add a type of natural numbers) Peano's axioms.

Following Russell, Henkin, and Prawitz, since we are assuming a primitive equality predicate at each type, we can define the usual logical connectives as in Figure 5 below.

$\begin{array}{llllll}\top & := & *=* & p \vee q & := & \forall_{x: \Omega}(((p \Rightarrow x) \wedge(q \Rightarrow x)) \Rightarrow x) \\ p \wedge q & := & \langle p, q\rangle=\langle\top, \top\rangle & \forall_{x: A} \varphi(x) & := & \{x: A \mid \varphi(x)\}=\{x: A \mid \top\} \\ p \Rightarrow q & := & p \wedge q=p & \exists_{x: A} \varphi(x) & := & \forall y: \Omega(\forall x: A((\varphi(x) \Rightarrow y) \Rightarrow y)) \\ \perp & := & \forall_{x: \Omega} x & \exists_{x: A} \varphi(x) & := & \exists x^{\prime}: A\left(\{x: A \mid \varphi(x)\}=\left\{x: A \mid x=x^{\prime}\right\}\right) \\ \neg p & := & \forall_{x: \Omega}(p \Rightarrow x) & & & \end{array}$

FIGURE 5. Type-theoretic encoding of logic

We now define an entailment relation $\Gamma \vdash_{X} q$. Here, $\Gamma$ is a finite set of formulas (i.e., terms of type $\Omega), q$ is a formula, and $X$ is a typed variable context containing all the free variables of $\Gamma$ and $q$; the

\footnotetext{
${ }^{9}$ Grothendieck toposes were introduced in the early 1960s by the Grothendieck school of algebraic geometry [4] as sheaves on a site. In the early 1970s, Lawvere and Tierney [80] introduced elementary toposes. It was realized that such toposes could be considered as a universe of mathematics, where the objects and morphisms can be treated as sets and functions, provided one refrains from using classical reasoning (the law of excluded middle and the Axiom of Choice).

${ }^{10}$ Moreover, as discussed in [77], strict logical functors will preserve only the powerset structure on the nose. In keeping with the logic literature and because of its historical importance, we denote the type of truth values by $\Omega$, rather than treating it as P1. Logical functors will preserve $\Omega$ on the nose.
} 
meaning of $\Gamma \vdash_{X} q$ is that $q$ can be derived (using the rules for intuitionistic logic) from $\Gamma$. When $\Gamma=\emptyset$ we simply write $\vdash_{X} q$. There are standard structural rules (including Cut), substitution, rules for equality, rules for products, and for comprehension. For example, there is the comprehension rule

$$
\vdash_{X}(y \in\{x: A \mid \varphi(x)\})=\varphi(y) .
$$

We refer to [77] for a complete list of rules.

By a type theory we mean an extension of HOL by sequents $\Gamma \vdash_{X} q$. When $\mathcal{L}$ is such a type theory, we write $\vdash^{\mathcal{L}}$ for the entailment relation of $\mathcal{L}$ (although we may omit the superscript when $\mathcal{L}$ is understood). In $\mathcal{L}$, we say that two terms $t, s$ of the same type are provably equal when $\vdash_{X}^{\mathcal{L}} t=s$. Just as for simply typed lambda calculi, it is common to include a type of natural numbers; the type theory obtained by adding the natural numbers to HOL (and no further basic types) is called Higher-order intuitionistic Arithmetic, or HAH for short.

An interpretation of one type theory in another is a mapping of types to types that preserves all type formation operations, together with a mapping of terms that respects the typing, the term formation operations and the provable equality. Type theories and interpretations form a category denoted Lang.

A type theory is classical if in addition it has Aristotle's axiom of excluded middle: $\forall p: \Omega(p \vee \neg p)$. Such a system of classical type theory was employed in Gödel's famous incompleteness paper [38].

Given a type theory $\mathcal{L}$ one may now build a syntactic topos as follows:

Definition 3.10 (Generated Toposes $T(\mathcal{L})$ ). The topos $T(\mathcal{L})$ generated by the type theory $\mathcal{L}$ has as objects "sets" (i.e., closed terms $\alpha$ of type P $A$, modulo provable equality). Morphisms $\alpha \rightarrow \beta$, where $\alpha: \mathrm{P} A$ and $\beta: \mathrm{P} B$, are "provably functional relations", i.e. closed terms $\varphi: \mathrm{P}(A \times B)$ (modulo provable equality) such that we can prove:

$$
\vdash^{\mathcal{L}} \forall_{x: A}\left(x \in \alpha \Rightarrow \exists !_{y: B}(y \in \beta \wedge(x, y) \in \varphi)\right)
$$

$T(\mathcal{L})$ is the category of "sets" and "functions" formally definable in higher-order logic $\mathcal{L}$.

The assignment $\mathcal{L} \mapsto T(\mathcal{L})$ is a functor $T:$ Lang $\rightarrow$ Top. For $\mathcal{L}_{0}=$ pure type theory, $T\left(\mathcal{L}_{0}\right)$ is

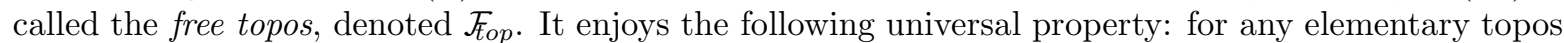
$\mathcal{E}$ there exists a logical functor $F: \mathcal{F}_{\text {top }} \rightarrow \mathcal{E}$ which is unique up to (unique) natural isomorphism. In other words, $\mathcal{F}_{\text {top }}$ is the initial object of Top.

In the other direction we may assign to a topos $\mathcal{C}$ its internal language $L(\mathcal{C})$, just as for CCCs. This gives a functor $L:$ Top $\rightarrow$ Lang.

Theorem 3.11 (Lambek-Scott [77]). The functors L, T described above constitute an equivalence of categories

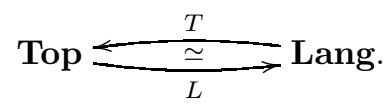

As for simply typed lambda calculus, we may extend this result by adding datatypes. Most importantly, we can consider type theories with natural numbers and toposes with natural number objects (see next Section).

\section{What aRe COMPUtABlE FUnCtions in CATEGORIES?}

We turn to the study of computable functions in categories. In this section, we limit ourselves to computable numerical functions; later we shall consider computable maps on other datatypes.

4.1. Natural Numbers Objects and Prim. In order to discuss number-theoretic functions in categories, we briefly recall Lawvere's notion of Natural Numbers Objects (NNOs) in cartesian closed categories [78, 77] and more generally NNOs in cartesian and monoidal categories [100]. 
Definition 4.1 (Lawvere [78]). A Natural Numbers Object (NNO) in a (cartesian closed) category $\mathcal{C}$ is a diagram $1 \stackrel{0}{\longrightarrow} \mathrm{N} \stackrel{S}{\longrightarrow} \mathrm{N}$ initial among diagrams $1 \stackrel{a}{\longrightarrow} A \stackrel{h}{\longrightarrow}$ A. i.e., there exists a unique $I t_{a h}: \mathrm{N} \rightarrow A$ satisfying:

$$
I t_{a h} \circ 0=a, I t_{a h} \circ S=h \circ I t_{a h}
$$

Existence, without uniqueness, of such an arrow $I t_{a h}$ yields the notion of a weak NNO. Any arrow $I t_{a h}$ : $\mathrm{N} \rightarrow A$ (unique or not) satisfying the equations above is called an iterator at type A. Diagrammatically,

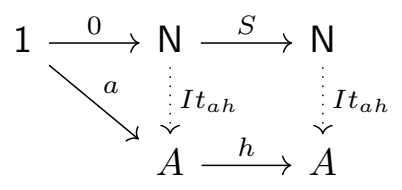

In Set this says:

$$
\begin{aligned}
& I t_{a h}(0)=a \\
& I t_{a h}(n+1)=h\left(I t_{a h}(n)\right)
\end{aligned}
$$

For any NNO (weak or strong) we may define, for any natural number $n \in \mathbb{N}$, the standard numeral $\widehat{n}: 1 \rightarrow \mathrm{N}$ by

$$
\widehat{0}=0 \quad \widehat{n+1}=S \circ \widehat{n} .
$$

We stress that depending on the nature of the ambient category, there may be non-standard numerals, that is, points $1 \rightarrow \mathrm{N}$ that are not of the form $\widehat{n}$.

A natural numbers object in a cartesian closed category is equivalent to the following scheme of Iteration with parameters. This general scheme (and its variants for monoidal categories) is sufficient for representing the primitive recursive functions [78, 77] and is the appropriate definition for NNO's in cartesian (or monoidal) categories, as in [77], p.71.

Definition 4.2 (Parametrized NNO). A diagram $1 \stackrel{0}{\longrightarrow} \mathrm{N} \stackrel{S}{\longrightarrow} \mathrm{N}$ in a cartesian category $\mathcal{C}$ is a parametrized NNO if for all arrows $A \stackrel{g}{\longrightarrow} B, B \stackrel{f}{\longrightarrow} B$, there exists a unique $I t_{g f}: \mathrm{N} \times A \rightarrow B$ such that:

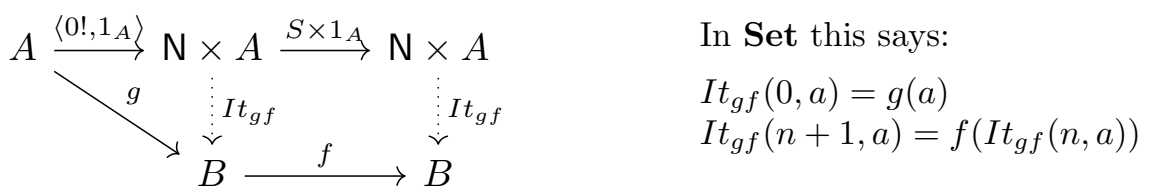

Existence without uniqueness of the arrow $I t_{g f}$ above yields a weak parametrized NNO.

A typical example is the notion of adding an iterator to a simply typed lambda calculus.

Example 4.3. Iterators in typed lambda calculus Following [77], we add to the terms of simply typed lambda calculus in Figure 3 an atomic type $\mathrm{N}$ and term formation operations

$$
0: \mathrm{N} \quad \frac{x: \mathrm{N}}{\mathrm{S} x: \mathrm{N}} \quad \frac{a: A \quad h: A^{A} \quad x: \mathrm{N}}{\operatorname{lt}(a, h, x): A}
$$

(allowing in particular the definition of standard numerals $\widehat{n}$ ). We then add to the equations of the simply typed lambda calculus the following equations:

$$
\operatorname{It}(a, h, 0)=_{X} a \quad \operatorname{It}(a, h, \mathrm{~S} x)={ }_{X \cup\{x\}} h(\operatorname{It}(a, h, x)), \text { provided } x \notin X .
$$

Calling this lambda theory $\mathcal{L}$, the associated syntactic category $C(\mathcal{L})$ (Definition 3.6) is a cartesian closed category with weak NNO.

In general, when we consider a category $\mathcal{C}$ the difference between a weak and a strong NNO in $\mathcal{C}$ can be understood in logical terms by considering the form of induction allowed in the internal language. For example, when $\mathcal{C}$ has a strong $\mathrm{NNO}$ we can prove the entailment

$$
\vdash_{x, y} x+y=y+x
$$


where, crucially $x, y$ are free variables of type $\mathrm{N}$. When $\mathcal{C}$ only has a weak $\mathrm{NNO}$ one can prove by (external) induction that for every $n \in \mathbb{N}$ :

$$
\vdash_{x} x+\widehat{n}=\widehat{n}+x
$$

Next, consider a (not necessarily symmetric) monoidal category $(\mathcal{C}, \otimes, I)$. Following Paré and Román [100], we may define notions of Left and Right NNOs, in analogy with Definition 4.2.

Definition 4.4 (Left Parametrized NNO). A diagram $I \stackrel{0}{\longrightarrow} \mathrm{N} \stackrel{S}{\longrightarrow} \mathrm{N}$ in a monoidal category $\mathcal{C}$ is a left parametrized $N N O$ if for all arrows $A \stackrel{g}{\longrightarrow} B, B \stackrel{f}{\longrightarrow} B$, there exists a unique $k: \mathrm{N} \otimes A \rightarrow B$ such that:

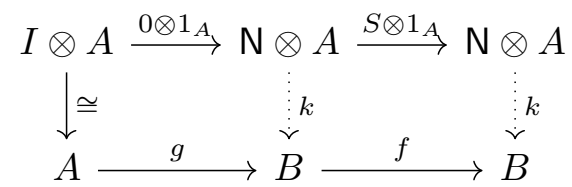

In the same manner, tensoring by $A$ on the left (rather than the right) results in a Right Parametrized $N N O$; weak objects are defined similarly by assuming merely existence (but not necessarily uniqueness) of $k$. For many examples of such monoidal NNOs, see [100].

We remark that there are yet other axiomatizations. A Peano-Lawvere category is a category for which the forgetful functor $\mathcal{C}^{\mathbb{N}} \rightarrow \mathcal{C}$ has a left adjoint (where $\mathbb{N}$ is regarded as the free monoid on one generator). A systematic study of the free such category can be found in Burroni's [12].

Another relevant class of categories is that of list-arithmetic pretoposes. These were developed by Maietti [89] (see also [90]) in order to provide a categorical setting accommodating Joyal's arithmetic universes ([56]), which in turn serve as a categorical account of the Incompleteness Theorem. A pretopos is a category that has finite limits, pullback-stable disjoint coproducts, and pullback-stable quotients of equivalence relations. Such a category has parameterized list objects when for each object $A$ there is an object $L A$ equipped with maps $e: 1 \rightarrow L A, c: L A \times A \rightarrow L A$ (thought of as the empty list and concatenation). These are required to satisfy the following universal property: for any $a: B \rightarrow C$ and $h: C \times A \rightarrow C$ there is a unique $I t_{a h}: B \times L A \rightarrow C$ making the following commute:

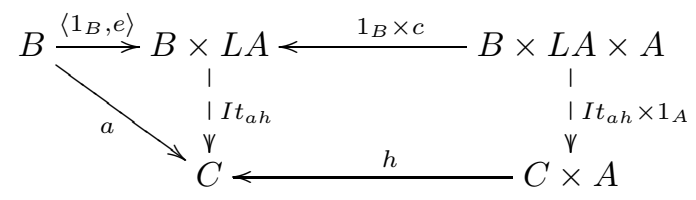

As is the case for NNOs, we may also consider a weak version where we only require existence and not uniqueness of the iterator $I t_{a h}$. A list-arithmetic pretopos is now defined as a pretopos admitting parametrized list objects for all $A$. Note that taking $A=1$ gives the notion of a parameterized NNO.

4.2. Representability. We now turn to representability of numerical functions in categories with NNOs.

Definition 4.5 (Lambek-Scott [77]). Let $\mathcal{C}$ be a cartesian category with a weak parametrized NNO $1 \stackrel{0}{\longrightarrow} \mathrm{N} \stackrel{S}{\longrightarrow} \mathrm{N}$. A function $f: \mathbb{N}^{k} \rightarrow \mathbb{N}$ is representable in $\mathcal{C}$ if there is an arrow $F: \mathrm{N}^{k} \rightarrow \mathrm{N} \in \mathcal{C}$ such that $F\left\langle\widehat{n_{1}}, \cdots, \widehat{n_{k}}\right\rangle=\widehat{m}$ whenever $f\left(n_{1}, \cdots, n_{k}\right)=m$.

Of course, the determination of which numerical functions are representable depends on the category: in the category Set, all numerical functions are representable! Following Lambek's question in the Introduction, we shall look at free categories with NNOs.

Theorem 4.6 (Román [108]). The class of representable numerical functions in $\mathcal{F}_{\text {cart }}$, the free cartesian category with parametrized NNO, is Prim. 
Hence the unique representation functor $\mathcal{F}_{\text {cart }} \longrightarrow$ Set has as image the subcategory of sets whose objects are powers $\mathbb{N}^{n}$ and whose maps are tuples of primitive recursive functions.

Román's proof essentially shows that Goodstein's development [40] of Skolem's primitive recursive arithmetic can be mimicked in $\mathcal{F}_{\text {cart }}$. In that sense, the result is not so surprising. However the following striking result considers the extension to $\mathcal{F}_{\text {mon }}$, the free monoidal category with a LNNO. Recall primitive recursion requires projection functions $U_{i}^{n}: \mathbb{N}^{n} \rightarrow \mathbb{N}$, yet in a monoidal category, in general $\otimes$ does not have explicit projections. Nevertheless:

Theorem 4.7 (Paré-Román [100]).

(i) The primitive recursive functions are representable in any monoidal category with LNNO.

(ii) Indeed, $\mathcal{F}_{m o n}$, the free monoidal category with LNNO, exists and is isomorphic to $\mathcal{F}_{\text {cart }}$, the free cartesian category with parametrized NNO.

Why is this? The reason is that the objects of $\mathcal{F}_{m o n}$ are generated from $\mathrm{N}$ under tensoring and we can code projections and diagonals between tensor powers $\mathrm{N} \otimes k$. This then allows the representability of the primitive recursive functions in a similar manner to $\mathcal{F}_{\text {cart }}$. The former result (coding projections and diagonals) is proved by an elegant categorical argument in Paré-Román, while Plotkin [104] gives a direct (albeit nontrivial) coding of the primitive recursive functions in $\mathcal{F}_{m o n}$.

4.3. Going beyond the primitive recursive functions: free CCCs. How do we get more representable functions? We increase the logical strength (the types) from the logic of $\{\wedge, \top\}$ (or $\{\otimes, I\}$ ) to the logic of $\{\wedge, \Rightarrow, \top\}$, i.e. to the cartesian closed level. Consider the free CCC with natural numbers generated by the empty graph, denoted $\mathcal{F}_{c c c}$ (as defined in Section 3.3).

The following is a theorem about simply typed lambda calculus, translated into the language of CCCs:

Theorem 4.8 (Lambek-Scott [77]). In $\mathcal{F}_{c c c}$, the free CCC with weak NNO:

(1) All primitive recursive functions and the Ackermann function are representable.

(2) The representable functions form a proper subclass of the total recursive functions, namely the provably total functions of Peano Arithmetic, or equivalently the $\varepsilon_{0}$-recursive functions [114, 37].

More generally, the representable total functions of $\mathcal{F}_{c c c}$ are the lowest level of the hierarchy of Gödel's Dialectica Functionals, i.e., Gödel's primitive recursive functionals of finite type [39, 113].

There is also a version of Gödel's Incompleteness for $\mathcal{F}_{c c c}$. Let $Z$ represent the zero function.

Theorem 4.9 (A version of Incompleteness, or 1 is not a generator). In $\mathcal{F}_{c c c}$, there is a closed term $F: \mathrm{N} \Rightarrow \mathrm{N}$ such that for each numeral $\widehat{n}, \vdash F \widehat{n}=\widehat{0}$, but $\forall F=Z$.

For a proof, see Corollaries 2.11, 2.12 in [77], p.263.

Finally, a topic of considerable importance in theoretical computer science:

Example 4.10. Computation by normalization We should also recall the notion of computation by normalization or, for a logician, by cut-elimination [37]. In the rewriting theory of typed lambda calculus, we can set up strongly normalizing rewrite systems in which terms can be rewritten to (unique) normal forms.

Given a term $f: \mathrm{N} \Rightarrow \mathrm{N}$ and a numeral $\widehat{n}: \mathrm{N}$, to compute $f \widehat{n}$ by normalization, we first normalize this term to its unique normal form of type $\mathrm{N}$. This yields a numeral $\widehat{m}$, for which we can prove $\vdash f \widehat{n}=\widehat{m}$; cf. [37]. This gives the value of $f$ on input numerals.

By Curry-Howard-Lambek, normalization techniques may also be used to solve coherence problems (decidability of equality) for various free CCCs, via their internal languages [77]: to check if two arrows in a free $\mathrm{CCC}$ are equal or not, it suffices to show that their normal forms (qua lambda terms) are identical, up to change of bound variables. 
4.4. Some properties of the free topos. Pure intuitionistic type theory $\mathcal{L}_{0}$ has many interesting properties, which translate into algebraic properties of the free topos $\mathcal{F}_{\text {top }}$ (see [77]) and are often key metamathematical principles of intuitionistic systems ([113]). In what follows we write $\vdash \operatorname{instead~of~} \vdash \mathcal{L}_{0}$ for derivability in intuitionist higher order arithmetic HAH.

- Consistency: not $(\vdash \perp)$.

- Disjunction Property: If $\vdash p \vee q$, then $\vdash p$ or $\vdash q$.

- Existence Property (EP): If $\vdash \exists_{x: A} \varphi(x)$ then $\vdash \varphi(a)$ for some closed term $a: A$.

In particular, in $\mathcal{F}_{\text {top }}$ EP says that numerals are standard, i.e. that numerals $1 \stackrel{f}{\longrightarrow} \mathrm{N}$ are all of the form $\widehat{n}$, for some $n \in \mathbb{N}$.

- Troelstra's Uniformity Principle (UP) for $A=\mathrm{P} C$ :

If $\vdash \forall_{x: A} \exists_{y: \mathrm{N}} \varphi(x, y)$ then $\vdash \exists_{y: \mathrm{N}} \forall_{x: A} \varphi(x, y)$.

In $\mathcal{F}_{\text {top }}$, UP says the arrows $\mathrm{PC} \rightarrow \mathrm{N}$ are constant, i.e. factor through a standard numeral.

- Independence of premisses (IP): If $\vdash \neg p \Rightarrow \exists_{x: A} \varphi(x)$ then $\vdash \exists_{x: A}(\neg p \Rightarrow \varphi(x))$.

- Markov's Rule (MR): If $\vdash \forall_{x: A}(\varphi(x) \vee \neg \varphi(x))$ and $\vdash \neg \forall_{x: A} \neg \varphi(x)$, then $\vdash \exists_{x: A} \varphi(x)$.

- The Existence Property with a parameter of type $A=\mathrm{P} C$ : If $\vdash \forall x: A \exists_{y: B} \varphi(x, y)$ then $\vdash \forall_{x: A} \varphi(x, \psi(x))$, where $\psi(x): B$.

Proofs: The original proofs [76] for EP and DP used Friedman (impredicative) realizability. When the authors lectured on this, Peter Freyd realized these rules had purely algebraic statements, with direct categorical proofs, using Artin gluing categories ([118]). The Freyd gluing techniques were expanded to include the proof rules above in [76] and in a series of later papers by the authors. This is also presented in $[77]$.

The free Boolean topos $\mathcal{F}_{b o o l}$ is defined in the same way as the free topos, but generated from classical type theory. As argued in [77], alas the free Boolean topos is not an ideal universe for classical mathematicians. For example, as a consequence of Gödel's Incompleteness Theorem, there are nonstandard numerals. To see this, let $G$ be any undecidable Gödel sentence. It may be shown that $\varphi(x):=(x=0 \Rightarrow G) \wedge(x \neq 0 \Rightarrow \neg G)$ determines a numeral $f: 1 \rightarrow \mathrm{N}$ in $\mathcal{F}_{b o o l}$; however, it cannot be a standard numeral, else we could decide $G$.

We now turn to the matter of representable numerical functions in the free topos. First we recall the definition of representability of a function in $\mathrm{HAH}^{11}$ :

Definition 4.11 (Representability in HAH). A total function $f: \mathbb{N}^{k} \rightarrow \mathbb{N}$ is representable in HAH when there exists a formula $R_{f}\left(x_{1}, \ldots, x_{k}, y\right)$ such that

(i) $f\left(n_{1}, \ldots, n_{k}\right)=m$ if and only if $\vdash R_{f}\left(\widehat{n_{1}}, \ldots, \widehat{n_{k}}, \widehat{m}\right)$

(ii) $\vdash \forall x_{1}: \mathrm{N} \ldots x_{k}: \mathrm{N} \exists$ ! $y: \mathrm{N} . R_{f}\left(x_{1} \ldots, x_{k}, y\right)$.

In the literature, one often considers a weaker notion of representability, in which clause (i) remains, but (ii) above is replaced by

(ii') for all $n_{1}, \ldots, n_{k} \in \mathbb{N} . \vdash \exists ! y:$ N. $R_{f}\left(\widehat{n_{1}}, \ldots, \widehat{n_{k}}, y\right)$.

We refer to this weaker notion as numeralwise representability.

It follows that a total numerical function $\mathbb{N}^{k} \rightarrow \mathbb{N}$ is representable in HAH if and only if it is representable by an arrow $N^{k} \rightarrow N$ in the free topos. (See Prop. 3.1, p. 264 in [77] for details.)

Theorem 4.12 (Lambek-Scott [77]).

\footnotetext{
${ }^{11}$ The same definition works in other formal systems such as Peano Arithmetic.
} 
(i) In HAH (and hence in the free topos), every representable numerical function is recursive. In particular, the global sections functor $\mathcal{F}_{\text {top }}(1,-): \mathcal{F}_{\text {top }} \rightarrow$ Set sends morphisms $\mathrm{N}^{k} \rightarrow \mathrm{N}$ to recursive functions $\mathbb{N}^{k} \rightarrow \mathbb{N}$.

(ii) Not all total recursive functions so arise.

(The second part of the theorem can be established by means of a diagonal argument.) This of course leads to the question of which total recursive functions are representable in HAH. This is related to the representability of numerical functions in Girard's system $\mathcal{F}_{\omega}$, but we shall not pursue this here. We note that the situation changes radically if we consider classical type theory (the free Boolean topos).

Theorem 4.13 (Lambek-Scott [77]).

(i) The numeralwise representable functions in classical type theory are exactly the total recursive functions (Gödel).

(ii) In classical type theory, numeralwise representable functions coincide with representable ones (by a result of $V$. Huber-Dyson [51], detailed in [77]). Hence the representable numerical functions in classical type theory are exactly the total recursive functions.

Unfortunately, as we have seen, the free Boolean topos has non-standard numerals. Thus, the global sections functor from the free Boolean topos to Set in general sends arrows $\mathrm{N}^{k} \rightarrow \mathrm{N}$ to partial, rather than total, numerical functions. This suggests that the representability of partial functions may be at least as important as that of total functions. In fact, we shall see that even at the intuitionistic level, the theory becomes much smoother.

Definition 4.14. A partial function $f: \mathbb{N}^{k} \rightarrow \mathbb{N}$ is representable in $\mathrm{HAH}$ if there is a formula $R_{f}\left(x_{1}, \ldots, x_{k}, y\right)$ such that

(i) for all $n_{1}, \ldots, n_{k} \in \mathbb{N}, f\left(n_{1}, \ldots, n_{k}\right)$ is defined and equal to $m$ if and only if $\vdash R_{f}\left(\widehat{n_{1}}, \ldots, \widehat{n_{k}}, \widehat{m}\right)$

(ii) $\vdash \forall x: \mathrm{N}^{k} \forall y: \mathrm{N} \forall z: \mathbf{N} . R_{f}(x, y) \wedge R_{f}(x, z) \Rightarrow y=z$.

We now have the following characterization:

Theorem 4.15 (Lambek-Scott [77]). A partial numerical function is representable in HAH (i.e., in the free topos) if and only if it is partial recursive.

4.5. C-monoids and Untyped Lambda Calculi. As mentioned earlier, Church's untyped lambda calculus played a key role in the original development of computability theory, as well as modern programming language theory. It was Dana Scott in the late 1960s who pointed out that untyped lambda calculi may be considered as typed lambda calculi with one non-trivial type (up to isomorphism). This arose from his development of domain theory, the mathematical modelling of untyped lambda calculi and the semantics of programming languages [2]. An algebraic framework for this development is given in [77], pp. 93-114, which we now sketch. For some historical references, the reader can see [72, 110].

Recall, monoids are categories with one object. A monoid has a terminal object precisely when it is trivial. However, when we ignore the terminal object, we may formulate a notion of cartesian closure:

Definition 4.16 (Lambek-Scott [77]). A $C$-monoid is a monoid $\mathcal{M}$ with constants $\pi_{1}, \pi_{2}, \varepsilon$, unary operation $(-)^{*}$, and binary operation $\langle-,-\rangle$ satisfying the equations of a CCC without a terminal object: i.e. products, surjective pairing, $\beta, \eta$. Explicitly:

$$
\begin{gathered}
\pi_{1}\langle a, b\rangle=a \quad \pi_{2}\langle a, b\rangle=b \quad\left\langle\pi_{1} c, \pi_{2} c\right\rangle=c \\
\varepsilon\left\langle h^{*} \pi_{1}, \pi_{2}\right\rangle=h \quad\left(\varepsilon\left\langle k \pi_{1}, \pi_{2}\right\rangle\right)^{*}=k
\end{gathered}
$$

The following results illustrate how C-monoids relate to untyped lambda calculi and CCCs. They are an untyped variation of Theorem 3.7.

Theorem 4.17 (Lambek-Scott [77]). 
(i) There is a bijective correspondence between C-monoids and untyped lambda calculi with products and surjective pairing. ${ }^{12}$ This correspondence extends to an isomorphism between the category of C-monoids and the category of such untyped lambda calculi (cf. [77], p.106).

(ii) $C$-monoids correspond to CCC's generated by a non-trivial reflexive object $U$, i.e., an object $U ¥ 1$ satisfying $U^{U} \cong U \cong U \times U$. In this case, $\operatorname{End}(U)$ will be such a $C$-monoid (cf. [77], p.99). Without the $\eta$-rule, we would have $U \times U \triangleleft U, U^{U} \triangleleft U$ (cf. also [2],[6] ).

(iii) With respect to appropriate numeral systems (e.g. Church or Barendregt numerals (see [6], Sections $6.3,6.4)$, the computable functions in the free $C$-monoid are precisely the partial recursive functions (cf. [77], p.276.)

We remark that part (ii) of the above theorem uses an observation of D. Scott ([110],[77]), which says: if we form the idempotent splitting completion (Karoubi envelope) of a C-monoid, we obtain a CCC which is generated by a reflexive object $U$. There are precise senses in which all C-monoids are isomorphic to such CCCs ([77], p.99.) Since Church's untyped lambda calculus was an early foundation of computability theory, it is no surprise that the computable functions in the free C-monoid are precisely the partial recursive ones.

4.6. Plotkin's characterization of Kleene's $\mu$-recursion. We recall Lambek's Lemma [68], which is often used in denotational semantics. Given an endofunctor $T: \mathcal{C} \rightarrow \mathcal{C}$ we define a $T$-algebra as a map $T A \rightarrow A$. Maps of $T$-algebras are commutative squares

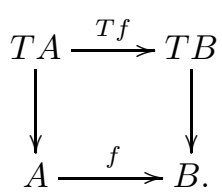

This gives a category of $T$-algebras; a $T$-algebra is called initial when it is an initial object in this category.

Lemma 4.18 (Lambek [68]). If $T A \stackrel{\alpha}{\longrightarrow} A$ is an initial T-algebra, then $\alpha$ is an isomorphism.

For us, the following is the prime example:

Example 4.19. The NNO $\mathbb{N}$ as an initial successor algebra in Set Consider the endofunctor $T(-)=$ $1+(-)$ on Set (often called the successor functor), with the $T$-algebra structure $(1+\mathbb{N}) \stackrel{\alpha}{\longrightarrow} \mathbb{N}$, where $\alpha=[0, S]$, for $1 \stackrel{0}{\longrightarrow} \mathbb{N}$ and $\mathbb{N} \stackrel{S}{\longrightarrow} \mathbb{N}$. The NNO property says that $\alpha$ is an initial $T$-algebra.

In Set, Lambek's Lemma then gives the familiar fact that $1+\mathbb{N} \stackrel{\alpha}{\longrightarrow} \mathbb{N}$ is an isomorphism, for $\alpha=[0, S]$. As we have seen above, initiality of $\alpha$ gives us primitive recursion. Now what about if we turn things around? Plotkin asked for the finality of the co-algebra $\alpha^{-1}: \mathbb{N} \rightarrow 1+\mathbb{N}$ - not in Set but in Par. Interestingly, this turns out to give exactly Kleene $\mu$-recursion for partial functions.

Let $\mathcal{C}$ be a monoidal category with (right distributive) binary sums and a weak left (or right) natural numbers object $I \stackrel{0}{\longrightarrow} \mathrm{N} \stackrel{S}{\longrightarrow} \mathrm{N}$. Following Plotkin, we extend Definition 4.5 of representable function to include partial functions, as follows. We shall say a partial function $f: \mathbb{N}^{k} \rightarrow \mathbb{N}$ is representable by an arrow $F: \mathrm{N}^{k} \rightarrow \mathrm{N} \in \mathcal{C}$ if for all $n_{1}, \ldots, n_{k} \in \mathbb{N}^{k}$,

$$
f\left(n_{1}, \cdots, n_{k}\right) \cong m \Rightarrow F\left\langle\widehat{n_{1}}, \cdots, \widehat{n_{k}}\right\rangle=\widehat{m}
$$

where $\cong$ means Kleene equality.

Theorem 4.20 (Plotkin [104]). Let $\mathcal{C}$ be a monoidal category with (right distributive) binary sums and a weak left (or right) natural numbers object $I \stackrel{0}{\longrightarrow} \mathrm{N} \stackrel{S}{\longrightarrow} \mathrm{N}$ such that $[0, S]$ is an isomorphism

\footnotetext{
${ }^{12}$ Such untyped lambda calculi extended with surjective pairing do not enjoy good rewriting properties. By a famous result of Klop [6], Ch.15, $\S 3$, the Church-Rosser theorem fails for them. Thus, the consistency of such systems would involve constructing a non-trivial C-monoid (cf. [77], pp.107-114.) or more general models [6].
} 
and $\left(\mathrm{N},[0, S]^{-1}\right)$ is a weakly final natural numbers coalgebra. Then all partial recursive functions are representable.

It is natural to ask if we can replace the " $\Rightarrow$ " in equation $(\dagger)$ above by the stronger condition " $\Leftrightarrow$ " (as in Definition 4.14 (i))? Plotkin calls this latter notion strong representability. The proof of Theorem 4.15 above (in [77], p.270) shows that for many arithmetical theories, representable partial functions are partial recursive. Plotkin takes the analog of this result (for strong representability) as an actual assumption to obtain a positive answer:

Theorem 4.21 (Plotkin [104]). Let $\mathcal{C}$ be a monoidal category with (right distributive) binary sums and a weak left (or right) natural numbers object $I \stackrel{0}{\longrightarrow} \mathrm{N} \stackrel{S}{\longrightarrow} \mathrm{N}$ such that $[0, S]$ is an isomorphism and $\left(\mathrm{N},[0, S]^{-1}\right)$ is a weakly final natural numbers coalgebra. If $0 \neq S 0$ and if all strongly representable functions are partial recursive, then all partial recursive functions are strongly representable in $\mathcal{C}$.

\section{Abstract Computability}

In this section we address the question: what is a category of computable maps? This should be compared with "synthetic" approaches to other areas of mathematics such as synthetic differential geometry, synthetic domain theory, homotopy type theory, and differential categories. A synthetic approach to computability aims at describing the categorical structure common to all reasonable notions of computation; hence in such categories every morphism is by definition computable. Note the contrast with the work described in the previous section, where one starts with a category that, a priori, has no prescribed computability-theoretic content, and where one identifies some maps as representing computable numerical functions.

Most notions of computation are inherently partial, in the sense that they allow for the computable maps to be partial maps. This fact, together with the importance of partial maps in other areas of mathematics, has resulted in a long history of studying partial maps in categories, going back to the early days of topos theory. This history largely overlaps with attempts to formulate aspects of computability theory in categorical terms, which in turn are closely related to the study of categories of domains, as in [2].

5.1. Categories of Partial Maps. We begin with a recent abstract treatment of categories of partial maps by Cockett and Lack [25]. There are at least two reasons for favouring this axiomatization: first, it is sufficiently general, in that it subsumes all the previous treatments. Second, it is algebraic, in the sense that it identifies categories of partial maps as ordinary categories equipped with additional algebraic structure. This allows for the application of powerful techniques from categorical algebra. For a much more detailed presentation and comparison with other approaches, see loc. cit. and follow-ups.

Definition 5.1 (Restriction Category). A restriction category is a category $\mathcal{C}$ together with an assignment $\overline{()}: \operatorname{Hom}_{\mathcal{C}}(A, B) \longrightarrow \operatorname{Hom}_{\mathcal{C}}(A, A)$ mapping $f \longmapsto \bar{f}$ satisfying:

$$
\begin{array}{ll}
\text { R.1 } & f \bar{f}=f \\
\text { R.2 } & \bar{f} \bar{g}=\bar{g} \bar{f} \text { whenever } \operatorname{dom}(f)=\operatorname{dom}(g) \\
\text { R.3 } & \overline{g \bar{f}}=\bar{g} \bar{f} \text { whenever } \operatorname{dom}(f)=\operatorname{dom}(g) \\
\text { R.4 } & \bar{g} f=f \overline{g f} \text { whenever } \operatorname{cod}(f)=\operatorname{dom}(g)
\end{array}
$$

We have $\overline{\bar{f}}=\bar{f}$, as well as $\bar{f} \bar{f}=\bar{f}$. Maps satisfying $f=\bar{f}$ are called restriction idempotents. The collection of restriction idempotents on $A$ is denoted $\mathcal{O}(A)$; the composition operation makes $\mathcal{O}(A)$ into a meet-semilattice; for each $f: A \rightarrow B$, there is an induced meet-semilattice homomorphism $f^{*}: \mathcal{O}(B) \rightarrow \mathcal{O}(A)$ sending $e \in \mathcal{O}(B)$ to $\overline{e f} \in \mathcal{O}(A)$. A map $f: A \rightarrow B$ is total if $\bar{f}=i d_{A}$. We obtain a wide subcategory $\operatorname{Tot}(\mathcal{C}) \hookrightarrow \mathcal{C}$.

Example 5.2. Examples of Restriction Categories 
(1) Par is a restriction category when we define

$$
\bar{f}(x)= \begin{cases}x & \text { if } x \in \operatorname{Dom}(f) \\ \uparrow & \text { else }\end{cases}
$$

(2) The restriction structure on Par is inherited by various subcategories, most notably the subcategory on the partial computable functions. This uses the fact that if $f$ is computable, then so is $\bar{f}$.

(3) Every category can be viewed as a restriction category by declaring $\bar{f}$ to be the identity for all $f$.

Definition 5.3 (Local Partial Order). For $f, g: A \rightarrow B$ in a restriction category, define

$$
f \leq g \quad \Leftrightarrow \quad f=g \bar{f} \text {. }
$$

For example, in Par, we have: $f \leq g$ precisely when $\operatorname{Graph}(f) \subseteq \operatorname{Graph}(g)$, i.e., when $g$ extends $f$.

Many notions for plain categories can be modified to make sense in the partial world. For example:

Definition 5.4 (Cartesian Structure). A restriction terminal object is an object 1 together with, for each object $A$, a unique total map $!_{A}: A \rightarrow 1$ with $!_{B} f \leq !_{A}$ for all $f: A \rightarrow B$.

A restriction product of $A, B$ is an object $A \times B$ with total projections $\pi_{A}, \pi_{B}$ such that for $f: C \rightarrow$ $A, g: C \rightarrow B$ there is a unique $\langle f, g\rangle$ with $\pi_{A}\langle f, g\rangle \leq f, \pi_{B}\langle f, g\rangle \leq g$ and $\overline{\langle f, g\rangle}=\bar{f} \bar{g}$.
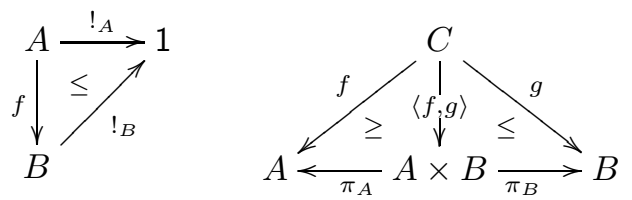

A Cartesian Restriction Category is a restriction category $\mathcal{C}$ which has a restriction terminal object and binary restriction products.

5.2. Turing Categories. Turing categories, introduced in [21] are restriction categories that essentially encode simultaneously the ideas underlying Kleene's $S_{n}^{m}$ and Enumeration theorems. They are also closely related to cartesian closed categories generated by models of untyped lambda calculus, in that they weaken the cartesian closure, while generalizing to the partial world.

Definition 5.5 (Turing Category). A Turing category is a cartesian restriction category $\mathcal{C}$ with an object $A$ (called a Turing Object), and a family of "universal application morphisms" $\left\{A \times X \stackrel{\tau_{X, Y}}{\longrightarrow} Y \mid X, Y \in \mathcal{C}\right\}$ with weak Currying: for every $Z \times X \stackrel{f}{\longrightarrow} Y$ there exists a total map $Z \stackrel{h}{\longrightarrow} A$ factoring through $\tau_{X, Y}$ :

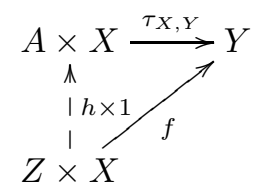

Note that this expresses the idea that $A$ acts as a weak exponential $Y^{X}$, for any pair of objects $X, Y$. One particular consequence is that every object is a retract of $A$. In particular, all finite restriction products $A^{n}$ are retracts of $A$.

An elementary but useful fact is the fact that the class of Turing categories is closed under idempotent splitting: if $\mathcal{C}$ is a Turing category, then so is $\mathcal{K}_{E}(\mathcal{C})$ where $E$ is the class of restriction idempotents.

A Turing category can equally well be described by "universal self-application" $\tau_{A A}$, denoted $A \times$ $A \stackrel{\bullet}{\longrightarrow} A$.

Theorem 5.6 (Cockett-Hofstra [21]). A Turing Category is a cartesian restriction category with an object $A$ such that (i) every object is a retract of $A$ and (ii) there is a universal self-application map $A \times A \stackrel{\bullet}{\longrightarrow} A$. 
Here are some of the motivating examples of Turing categories:

Example 5.7. Examples of Turing Categories

(1) Let $\left\{\phi_{m}\right\}_{m \in \mathbb{N}}$ be a standard enumeration of unary partial recursive functions (see [27]). Kleene's First Model $\operatorname{Comp}(\mathbb{N})$ is the category whose objects are powers $\mathbb{N}^{k}$ and whose maps $\mathbb{N}^{k} \rightarrow \mathbb{N}^{m}$ are $m$-tuples of partial computable functions of $k$ variables. $\mathbb{N}$ is a Turing object, there are retractions $\mathbb{N}^{k} \triangleleft \mathbb{N}$ and $m \bullet n:=\phi_{m}(n)$ gives a universal application, by Kleene's theorems. The restriction idempotents in this case are precisely the r.e. sets. Hence the restriction idempotent splitting of this category has the r.e. sets as objects, and partial computable functions as maps.

This example can be generalized to give categories $\operatorname{Comp}\left(\mathbb{N}^{A}\right)$, where $A$ is an oracle.

(2) Consider a C-monoid, or more generally a reflexive object $U$ in a ccc, where $1 \triangleleft U, U \times U \triangleleft U$, $U^{U} \triangleleft U$. If $(m, r): U^{U} \triangleleft U$ is a retraction pair, then $\bullet_{U}:=U \times U \stackrel{r \times i d_{U}}{\longrightarrow} U^{U} \times U \stackrel{e v}{\longrightarrow} U$ determines a total Turing structure with Turing object $\mathrm{U}$.

(3) Term models of Partial Combinatory Logic (PCL) yield Turing categories. PCL is an (partial) algebraic theory with constant symbols $\mathrm{s}, \mathrm{k}$ and one binary application symbol $\bullet$ (we write $x y$ instead of $x \bullet y$, and associate to the left). Terms are formed in the usual way, together with a clause for forming restricted terms:

$$
\text { Terms } \quad t, t^{\prime}::=\left.\operatorname{VAR}|\mathrm{s}| \mathrm{k}\left|t t^{\prime}\right| t\right|_{t^{\prime}}
$$

where $\left.t\right|_{t^{\prime}}$ is to be interpreted as " $t$ restricted to $d o m\left(t^{\prime}\right)$ ". (The categorical interpretation of such a restricted term is $\llbracket t \rrbracket \circ \overline{\left.\llbracket t^{\prime}\right]}$.) The following equations are imposed: $\mathrm{k} x y=x_{\mid y}, \mathrm{~s} x y z=x z(y z)$, and sxy $\downarrow$. See [23] for details.

The case of the closed term model is particularly significant because it corresponds to the initial Turing category. Note that a total point $t: 1 \rightarrow A$ of the Turing object corresponds to a provably total closed term $t$ of $P C L$. The global sections functor is therefore not faithful, since there exist many closed terms that are not provably total, for example $\left.\mathrm{k}\right|_{P}$ where $P$ is the paradox combinator.

From the axioms of a Turing category, one may derive some basic results from computability theory such as the recursion theorems. The restriction idempotents (partial identities serving as the domains of maps) in a Turing category play the role of recursively enumerable sets; pullback of restriction idempotents then corresponds to m-reducibility. Note that the standard model $\operatorname{Comp}(\mathbb{N})$ also has ranges, in the sense that every morphism not only has a domain but also a range; such categories are studied in detail in [19, 20]; see also [117].

Since the axioms of a Turing category do not preclude total models, one cannot expect results such as the undecidability of the halting problem or Rice's theorem to follow in general. A detailed discussion of the development of basic computability theory in the setting of Turing categories and how this depends on additional structure can be found in [18].

5.3. Computable maps and PCAs. Turing categories are closely related to a class of structures called partial combinatory algebras (PCAs), as suggested by Example 3.

Let $\mathcal{C}$ be a cartesian restriction category. An applicative structure in $\mathcal{C}$ is a pair $\mathcal{A}=(A, \bullet)$, where $A \times A \stackrel{\bullet}{\longrightarrow} A$ is a morphism called application. There are no requirements on $\bullet$ (such as associativity). Define $\bullet^{n}: A \times A^{n} \rightarrow A$ inductively, so $\bullet^{n+1}:=A \times A \times A^{n} \stackrel{\bullet \times i d}{\longrightarrow} A \times A^{n} \stackrel{\bullet^{n}}{\longrightarrow} A$.

Definition 5.8 (Computable maps). A map $A^{n} \stackrel{f}{\longrightarrow} A$ is $\mathcal{A}$-computable when it is "named" by a total point of $A$, i.e. there is a total point $p: 1 \rightarrow A$ such that (identifying $A^{n}$ with $1 \times A^{n}$ ):

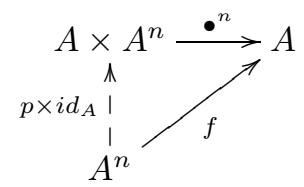


(Intuitively, $f(\vec{x})=p \bullet \vec{x}$.) Moreover, we require that $f$ is total on its first $n-1$ arguments. More generally, we say a map $f: A^{n} \rightarrow A^{m}$ is $\mathcal{A}$-computable if all its components are.

Since there are no axioms on an applicative object, the collection of $\mathcal{A}$-computable maps cannot be expected to have any good closure properties. In particular, it cannot be expected to form a subcategory of $\mathcal{C}$. When it does, the object $\mathcal{A}$ is called combinatory complete. This characterization is the categorical formulation of combinatory completeness (see also [86]).

Classically, an applicative structure is called combinatory complete when every "polynomial" built from variables, elements of $A$ and application, is represented by an element of $A$, see $[7,116]$. When $t$ is a polynomial and $x$ is a variable, we write $\lambda^{*} x$.t for the element representing $t$. That is: $\left(\lambda^{*} x . t\right) a=t[a / x]$ for all $a \in A$. Equivalently, an applicative structure is a PCA exactly when it is a model of the theory PCL (see Example 3 above).

Definition 5.9. A combinatory complete applicative structure $\mathcal{A}$ is called a partial combinatory algebra $(\mathrm{PCA})$. For $\mathcal{A}$ a $\mathrm{PCA}$, denote by $\operatorname{Comp}(\mathcal{A})$ the restriction category whose objects are the finite powers of $A$ and whose morphisms are the $\mathcal{A}$-computable maps.

At first sight, it may not be evident that combinatory completeness has many interesting consequences. It ensures, however, that PCAs are powerful enough to represent various useful programming constructs.

Lemma 5.10. In any $P C A$, we can define the following:

(i) Booleans, pairs, numerals (using e.g. $\widehat{n}=\lambda^{*} a \lambda^{*} f . f^{n} a$ ). What is more, any partial computable function $f: \mathbb{N} \rightarrow \mathbb{N}$ can then be represented in $\mathcal{A}$ in the sense that there is an element $a_{f} \in \mathcal{A}$ for which $f(n)=m$ implies $a_{f} \widehat{n}=\widehat{f(n)}$.

(ii) Fixed points: there is an element y $\in A$ for which y $=a(\mathrm{y} a)$ for all $a \in A$.

(iii) Recursors: there is an element $r \in A$ for which rab $\widehat{0}=a, \operatorname{rab}(\widehat{n+1})=b($ rab $\widehat{n}) \widehat{n}$ for all $a, b \in A$ and $n \in \mathbb{N}$.

Standard examples of PCAs include Kleene's first model (natural numbers with partial recursive application, typically denoted $\mathcal{K}_{1}$ ), term models of PCL, and models of untyped lambda calculus. The following example is of importance in higher-order computability, and will be used in the next section. We presuppose a surjective coding of finite sequences $\langle-\rangle: \mathbb{N}^{*} \rightarrow \mathbb{N}$. For $g: \mathbb{N} \rightarrow \mathbb{N}$, let $\hat{g}(k)=\langle g(0), \ldots, g(k-1)\rangle$. Finally, let $*$ denote concatenation of sequences; for a sequence $L$ and $n \in \mathbb{N}$ we write $n * L$ instead of $\langle n\rangle * L$.

Example 5.11. Kleene's second model Consider $f, g \in \mathbb{N}^{\mathbb{N}}$. Define a (possibly partial) function $f \star g$ : $\mathbb{N} \rightarrow \mathbb{N}$ by

$$
(f \star g)(n)= \begin{cases}f(\langle n * \hat{g}(k)\rangle)-1 & \text { where } k=\mu_{r} . f(\langle n * \hat{g}(r)\rangle)>0 \\ \text { undefined } & \text { if no such } k \text { exists. }\end{cases}
$$

Then define a partial application $\bullet: \mathbb{N}^{\mathbb{N}} \times \mathbb{N}^{\mathbb{N}} \rightarrow \mathbb{N}^{\mathbb{N}}$ by

$$
f \bullet g= \begin{cases}f \star g & \text { if } f \star g \text { is total } \\ \text { undefined } & \text { otherwise. }\end{cases}
$$

This model is typically denoted $\mathcal{K}_{2}$, and captures a notion of "computable operations acting on continuous data". If we restrict $\mathbb{N}^{\mathbb{N}}$ to the set of total computable functions, we get a sub-PCA $\mathcal{K}_{2}^{\text {eff }}$ of "computable operations acting on computable data". For details see [85].

What is the correct notion of morphism of PCAs? Regarding $\mathcal{A}, \mathcal{B}$ as computational devices, a morphism $\varphi: \mathcal{A} \rightarrow \mathcal{B}$ should at least express that $\mathcal{A}$ can be interpreted, or simulated, within $\mathcal{B}$. The following definition is due to Longley [82]. We state it in set-theoretic terms here, but it can easily be rendered diagrammatically in a cartesian restriction category:

Definition 5.12 (Simulation). A simulation from $\mathcal{A}$ to $\mathcal{B}$ is a function $\varphi: A \rightarrow B$ for which there exists $b \in B$ such that

$$
x \bullet y \downarrow \quad \Longrightarrow \quad b \bullet \varphi(x) \bullet \varphi(y)=\varphi(x \bullet y) .
$$


Simulations compose, and in fact form a 2-category. We point out that in [82] a relational version of this definition is given; however, as demonstrated in [50], it is possible to view relational simulations as Kleisli morphisms over a base category of functional simulations.

Example 5.13. Numerals as Simulation Every PCA admits a choice of numerals; such a choice amounts to a simulation $\mathcal{K}_{1} \rightarrow \mathcal{A}$. All non-constant simulations $\mathcal{K}_{1} \rightarrow \mathcal{A}$ are in fact isomorphic to each other.

Returning to the connections between PCAs and Turing categories, we note that by construction $\operatorname{Comp}(\mathcal{A})$ is a cartesian restriction category. The following shows that PCAs are a fundamental notion for generating Turing categories: every PCA gives rise to a Turing category, and every Turing category is generated by the PCA structure on the Turing object.

Theorem 5.14 (Cockett-Hofstra [21]).

(i) If $\mathcal{A}=(A, \bullet)$ is a $P C A$, then $\operatorname{Comp}(\mathcal{A})$ is a Turing category, with Turing object $A$.

(ii) If $(\mathcal{C}, A)$ is a Turing category with Turing object $A$, then $(A, \bullet)$ is a $P C A$ and $\mathcal{C} \cong \mathcal{K}_{E}(\mathbf{C o m p}(\mathcal{A}))$, for some class of idempotents $E$.

Thus "Categories of the form $\operatorname{Comp}(\mathcal{A})$ serve as a minimal environment (for) $P C A$ 's and ... computable maps ...; other Turing categories are supposed to be viewed as (non-essential) inflations of such minimal categories" ([21]).

Earlier we contrasted the approach of identifying representable numerical functions in free categories with NNO with the synthetic approach of Turing categories. However, there is a slightly different perspective on Turing categories, that perhaps brings the two approaches closer together.

Instead of considering Turing categories in isolation, i.e., synthetically, one can consider Turing categories structured over a base category. For example, the Turing category $\operatorname{Comp}(\mathbb{N})$ can be considered as a non-full subcategory of Par. This point of view is particularly relevant when one wishes to consider non-computable functions or study, e.g., non-r.e.degrees. More generally, we think of a Turing category $\mathcal{C}$ with a cartesian restriction functor $F: \mathcal{C} \rightarrow \mathcal{B}$ into a base category $\mathcal{B}$ as specifying an object $F A$ of $\mathcal{B}$ together with a notion of computation on $F A$. The object $F A$ is necessarily a PCA, but $\mathcal{C}$ is not always $\operatorname{Comp}(F A)$; the reason is that $F A$ may have total elements $t: 1 \rightarrow F A$ that are not in the image of $F$. Hence $\operatorname{Comp}(F A)$ may contain morphisms that are not represented in $\mathcal{C}$. This forces the consideration of relative $P C A s$, and the full characterization of Turing categories over a fixed base in terms of such relative PCAs can be found in [22]. ${ }^{13}$

Note that there is an analogy between the two perspectives on Turing categories and those on toposes: one may consider toposes relative to a fixed base topos $\mathcal{S}$ (as is common in the study of Grothendieck toposes, where $\mathcal{S}$ plays the role of the universe of sets), or one may study elementary toposes such as the free topos without regarding them as being constructed over a base.

\section{Realizability}

We now briefly turn our attention to a strand of research that also heavily involves the study of categorical structures associated to models of computation, but that is different from the earlier themes in that it primarily considers such structures as models of various logical systems.

6.1. Kleene Realizability. Realizability, originally devised by Kleene in the seminal paper $[60]^{14}$, is to be thought of as a semantics for constructive mathematical systems ${ }^{15}$. In Kleene's original work, the system at hand was Heyting Arithmetic (HA), and the interpretation was defined in terms of partial computable functions. The central notion is written $n \underline{\mathbf{r}} A$, where $n \in \mathbb{N}$ and $A$ a formula in the language of arithmetic, and is pronounced " $n$ realizes $A$ ", or " $n$ is a realizer for $A$ ". The intuition is that $n$ codes

\footnotetext{
${ }^{13}$ This characterization involves a notion of simulation between Turing categories (over a fixed base), generalizing the foundational work by Longley [82] on simulations between PCAs (called applicative morphisms in loc. cit.).

${ }^{14}$ We omit a discussion of the history of the subject, of which some of the main threads are detailed in [115].

${ }^{15}$ Recent work by Krivine and others has shown that it is also possible to define realizability interpretations of classical systems.
} 
information about why $A$ is true. The definition is by induction on the structure of $A$ (and uses an enumeration $\left.\phi_{0}, \phi_{1}, \ldots\right)$ of unary partial computable functions:

Definition 6.1 (Kleene Realizability). Define $n \underline{\mathbf{r}} A$ (for sentences $A$ ) by

$$
\begin{aligned}
& n \underline{\mathbf{r}} t=s \quad \text { iff } \quad n=0 \text { and } t=s \text { is true } \\
& n \underline{\mathbf{r}} A \wedge B \quad \text { iff } \quad n=\langle a, b\rangle \text { where } a \underline{\mathbf{r}} A \text { and } b \underline{\mathbf{r}} B \\
& n \underline{\mathbf{r}} A \vee B \quad \text { iff } \quad n=\langle a, b\rangle \text { where either } a=0 \text { and } b \underline{\mathbf{r}} A \text { or } a=1 \text { and } b \underline{\mathbf{r}} B \\
& n \underline{\mathbf{r}} A \rightarrow B \quad \text { iff } \quad \text { for all } m \in \mathbb{N} \text {, if } m \underline{\mathbf{r}} A \text { then } \phi_{n}(m) \downarrow \text { and } \phi_{n}(m) \underline{\mathbf{r}} B \\
& n \underline{\mathbf{r}} \exists x . A \quad \text { iff } \quad n=\langle a, b\rangle \text { where } b \underline{\mathbf{r}} A[a / x] \\
& n \underline{\mathbf{r}} \forall x . A \quad \text { iff } \quad \text { for all } m \in \mathbb{N}, \phi_{n}(m) \downarrow \text { and } \phi_{n}(m) \underline{\mathbf{r}} A[m / x]
\end{aligned}
$$

The Soundness theorem now states: $H A \vdash A \Longrightarrow \exists n \in \mathbb{N} . n \underline{\mathbf{r}} A$. The converse, however, is false: there are realizable statements that are underivable. Most notably, Extended Church's Thesis $\left(E C T_{0}\right)$ is the scheme:

$$
\forall x(A(x) \rightarrow \exists y . B(x, y)) \rightarrow \exists e \forall x(A(x) \rightarrow B(x, e \bullet x))
$$

Here, $A$ is assumed to be an almost negative formula, and $e \bullet x$ denotes the application of the $e$-th computable function to $x$, suitably represented in $H A$. One can show that all instances of $E C T_{0}$ are realizable but not provable in $H A$. Moreover, $E C T_{0}$ axiomatizes Kleene realizability, in the sense that the realizable statements of $H A$ are precisely those that are derivable in $H A+E C T_{0}$.

Over the years, many variations on Kleene's original definition have been studied, with the purpose of establishing, among other things, consistency results and proof-theoretic properties of various formal systems. For example, q-realizability incorporates derivability into the definition of realizability, and can be used to establish the existence and disjunction properties of HA.

6.2. Realizability Toposes. How does realizability manifest itself categorically? Historically, the topos-theoretic treatment of Boolean-valued and Heyting-valued models $([34,46])$ inspired the idea of considering sets of realizers as truth values. This idea led Hyland to the discovery of the Effective Topos [52], an elementary (non-Grothendieck) topos with the property that the first-order arithmetical statements about the NNO are precisely the Kleene-realizable statements. Thus, among other things, the internal language of $\mathbf{E f f}$ is a natural extension of Kleene realizability to higher-order logic.

Various notions from computability theory find a natural home in Eff. For example, the Turing degrees manifest themselves in the form of subtoposes of $\mathbf{E f f}$ :

Theorem 6.2 ([52, 102]). The lattice of Turing degrees faithfully embeds into the lattice of subtoposes of $\mathbf{E f f}$.

(Here, the notion of subtopos is taken in the geometric sense: it is a full subcategory closed under finite limits, whose inclusion has a finite-limit preserving left adjoint.) Not every subtopos arises from a Turing degree however; see [81] for more information.

There are several ways to present the Effective Topos and its variants. Perhaps the simplest is via exact completions (see [13, 16], as well as [92]). A category $\mathcal{C}$ is called exact if it has finite limits, pullback-stable quotients of equivalence relations, and if every coequalizer is the coequalizer of its kernel pair. Every topos is exact. Now to each category with finite limits $\mathcal{C}$ one may associate an exact category $\mathcal{C}_{\text {ex/lex }}$ by freely adding quotients of equivalence relations, and the Effective Topos is of this form. The finite limit category in question is called Pasm, the category of partitioned assemblies.

Definition 6.3 (Partitioned Assemblies). The category Pasm has objects pairs $(X, \alpha)$ with $X$ a set and $\alpha: X \rightarrow \mathbb{N}$ a function; a morphism $(X, \alpha) \rightarrow(Y, \beta)$ is a function $f: X \rightarrow Y$ which is tracked, in the sense that there exists a code $e \in \mathbb{N}$ such that

$$
\forall x \in X . e \bullet \alpha(x) \downarrow \wedge e \bullet \alpha(x)=\beta(f(x)) .
$$

Theorem 6.4 (Carboni et al. [13,14]). The Effective Topos is the exact completion of the category of partitioned assemblies: $\mathbf{E f f} \simeq \mathbf{P a s m}_{e x / l e x}$. 
The above construction of Eff can be refined by considering an intermediate category:

Definition 6.5 (Assemblies). The category Asm has objects pairs $(X, \alpha)$ with $X$ a set and $\alpha: X \rightarrow$ $\mathcal{P}_{+} \mathbb{N}$ a function (where $\mathcal{P}_{+}$denotes the non-empty powerset); a morphism $(X, \alpha) \rightarrow(Y, \beta)$ is a function $f: X \rightarrow Y$ which is tracked, in the sense that there exists a code $e \in \mathbb{N}$ such that

$$
\forall x \in X \forall a \in \alpha(x) . e \bullet a \downarrow \wedge e \bullet a \in \beta(f(x)) .
$$

The category Asm is regular: it has finite limits and admits stable quotients of equivalence relations. Any finite limit category $\mathcal{C}$ admits a free regular completion $\mathcal{C}_{\text {reg }}$, and any regular category $\mathcal{D}$ admits a free exact completion $\mathcal{D}_{\text {ex/reg }}$. With this notation, we now have the following relations between Pasm, Asm, and Eff:

Theorem 6.6 (Carboni et al. [13, 14]). There are equivalences $\mathbf{A s m} \simeq \mathbf{P a s m}_{\text {reg }}$ and $\mathbf{E f f} \simeq \mathbf{A s m}_{\text {ex } / r e g}$.

The category of assemblies happens to be much more than regular: it is a quasitopos and has a NNO, given by $(\mathbb{N},\{-\})$. As such, a lot of the computability-theoretic features of Eff already manifest themselves in this subcategory. For example, in Asm we may consider higher-type computability over $\mathbb{N}$.

An alternative construction of Eff, more logical in nature, makes use of the concept of a tripos (see [53]; tripos is an acronym for "topos-representing indexed preordered set".) One considers the Set-indexed preorder $\operatorname{Set}(-, \mathcal{P} \mathbb{N})$; for a set $X$, we preorder $\operatorname{Set}(X, \mathcal{P} \mathbb{N})$ by:

$$
\alpha \vdash_{X} \beta \Longleftrightarrow \exists e \in \mathbb{N} \forall x \in X \forall a \in \alpha(x) . e \bullet a \downarrow \wedge e \bullet a \in \beta f(x) .
$$

There is now a general construction turning a tripos into a topos, and Eff arises as the result of applying this construction to $\operatorname{Set}(-, \mathcal{P N})$. This construction highlights the original idea of regarding sets of realizers as truth-values, in analogy with $H$-valued sets for $H$ a complete Heyting algebra.

6.3. PCAs and Toposes. The construction of the Effective Topos generalizes in various ways. We focus on the following fact ${ }^{16}$ : for each $\mathrm{PCA} \mathcal{A}=(A, \bullet)$, there is an associated realizability topos $\mathbf{R T}(\mathcal{A})$. In fact, we may associate to $\mathcal{A}$ a category of partitioned assemblies $\operatorname{Pasm}(\mathcal{A})$ (where the objects are sets $X$ equipped with a function $\alpha: X \rightarrow A)$, and let $\operatorname{RT}(\mathcal{A})=\operatorname{Pasm}(\mathcal{A})_{\text {ex } / \text { lex }}$. Alternatively we build the tripos $\operatorname{Set}(-, \mathcal{P} A)$. The functoriality of $\mathcal{A} \mapsto \mathbf{R T}(\mathcal{A})$, including the correct notion of "Morita equivalence" for PCAs was worked out in [82]; the complete characterization of (geometric) morphisms between toposes of the form $\mathbf{R T}(\mathcal{A})$ in terms of morphisms of (ordered) PCAs appears in [50].

An important construction, both for the analysis of realizability toposes and for applications of realizability, is that of the category of PERs over a PCA. A PER (partial equivalence relation) on a set $A$ is simply a symmetric and transitive relation; equivalently, it is an equivalence relation on a subset of $A$ (then called the domain of the PER). When $R$ is a PER on $A$, we write $A / R=\{[a] \mid(a, a) \in R\}$ for the set of equivalence classes. In case of a PCA, this leads to the following:

Definition 6.7 (Category of PERs). Let $\mathcal{A}=(A, \bullet)$ be a PCA. The category $\mathbf{P E R}(\mathcal{A})$ has as objects PERs $(A, R)$ on $A$. A morphism $(A, R) \rightarrow(A, S)$ is a function $f: A / R \rightarrow A / S$ that is tracked in the sense that there exists $a \in A$ such that

$$
\forall x \in A .(x, x) \in R \rightarrow a \bullet x \downarrow \wedge f[x]=[a \bullet x] .
$$

The category $\operatorname{PER}(\mathcal{A})$ can be seen as a full subcategory of $\operatorname{Asm}(\mathcal{A})$ on those objects $(X, \alpha)$ for which $\alpha(x) \cap \alpha(y) \neq \emptyset$ implies $x=y$. It is (locally) cartesian closed, and has a NNO. We will return to this structure in the section on higher type computability below.

Since PCAs give rise both to Turing categories and to realizability toposes, it is natural to wonder how the latter two are related. We mention here one result that builds on earlier insights into how realizability toposes can be regarded as colimit completions [106, 107]. In [22] a universal property of

\footnotetext{
${ }^{16}$ It was already known well before the discovery of the effective topos that combinatory algebras carried sufficient structure to define notions of realizability, see e.g. [33].
} 
partitioned assemblies is exhibited: it is the free fibred preorder on a functor, in a suitable restrictioncategory theoretic sense. In case of a $\mathrm{PCA} \mathcal{A}$ with associated Turing category $\operatorname{Comp}(\mathcal{A})$, applying this construction gives a fibration, and taking total maps recovers $\operatorname{Pasm}(\mathcal{A})$. Moreover, this construction has the property that it turns simulations between Turing categories into actual functors on the level of partitioned assemblies.

To conclude our discussion of realizability toposes we mention the abstract characterization of toposes of the form $\mathbf{R T}(\mathcal{A})$ due to Frey [35]. In order to state this result, we need to define a few concepts. First, suppose that $\Gamma \dashv \nabla$ is a pair of adjoint functors with $\Gamma \circ \nabla \cong 1$. Then a map $f$ is called closed (w.r.t. this adjunction) if the square

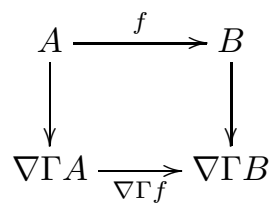

in which the vertical maps are the unit morphisms is a pullback ${ }^{17}$. Moreover, an object $A$ is called separated when the unit $A \rightarrow \nabla \Gamma A$ is monic ${ }^{18}$. Finally, an object is called discrete when it is orthogonal to all closed regular epimorphisms.

Theorem 6.8 (Frey [35]). A locally small category $\mathcal{E}$ is equivalent to $\mathbf{R T}(\mathcal{A})$ for a PCA $\mathcal{A}$ if and only if the following conditions hold:

- $\mathcal{E}$ is exact and locally cartesian closed;

- $\mathcal{E}$ has enough projectives and the full subcategory $\operatorname{Proj}(\mathcal{E})$ on the projective objects is closed under finite limits;

- The global sections function $\Gamma: \mathcal{E} \rightarrow$ Set has a right adjoint $\nabla$ which factors through $\operatorname{Proj}(\mathcal{E})$;

- There exists a separated, projective object $D$ such that for any projective object $P$ there exists a closed map $P \rightarrow D$.

This theorem should be regarded as the analogue of the well-known Giraud theorem characterizing Grothendieck toposes among exact categories in terms of their relation to Set. Note that the first conditions express that $\mathcal{E}$ is of the form $\mathcal{C}_{e x / \text { lex }}$, and that the last two conditions therefore characterize categories of the form $\operatorname{Pasm}(\mathcal{A})$.

We end this section by a brief mention of another approach to partial recursive functions and PER, introduced by Lambek [74] and studied further in [75]. In this view, one considers the category of relations generated by the monoid of primitive recursive functions (qua relations). Taking this viewpoint, a partial recursive function is simply a single-valued recursively enumerable (r.e.) relation, and the category PER is a kind of Karoubi envelope construction: the category whose objects are arbitrary pers on $\mathbb{N}$ and whose maps are r.e. functional relations between them. The full subcategory of PER given by r.e. pers and r.e. functional relations is particularly interesting in this regard, since it turns out to be exact. In [75], it is considered as a candidate for a kind of exact completion of the monoid of primitive recursive functions, although the precise nature of this completion is yet to be determined.

\section{Other Directions}

This final section briefly introduces some facets of computation that have a somewhat different character than the work discussed so far. First, we discuss traced monoidal categories and PCAs arising in "Geometry of Interaction" situations. Next, we turn to computability at higher type, giving a very brief introduction to some of the concepts and ideas in that area. Finally, we mention some of the categorical approaches to complexity theory.

\footnotetext{
${ }^{17}$ The terminology closed derives from the fact that for realizability toposes $\mathbf{R T}(\mathcal{A})$, closed subobjects for the double negation topology are characterized by this condition.

${ }^{18}$ This terminology also derives from the fact that in $\operatorname{RT}(\mathcal{A})$ this characterizes the separated objects for the double negation topology.
} 
7.1. Traced Categories. In an influential paper, Joyal, Street, and Verity [57] introduced the notion of an abstract trace in monoidal categories. Such traces arise in a wide range of areas, including knot theory, fixed point theory and theoretical computer science. We will be especially concerned with applications arising in the algebra of feedback in networks and the associated fixed point theories. Traced monoidal categories also play a prominent role in the categorical analysis of Girard's Geometry of Interaction (GoI) Program in Linear Logic, in which one analyzes the dynamics and flow of information in cut-elimination in networks of proofs [1, 42]. For simplicity, we consider the case of symmetric monoidal categories.

A parametrized trace on a symmetric monoidal category $\mathcal{C}$ is an operation $\operatorname{tr}: \mathcal{C}(X \otimes U, Y \otimes U) \rightarrow$ $\mathcal{C}(X, Y)$, satisfying a number of axioms discussed in detail in [57, 1]. The theory has a particularly geometric flavour, and the papers, loc. cit., use a string calculus both for describing the axioms and for diagrammatic reasoning. A particular evocative picture is to think of the trace as a form of "feedback":

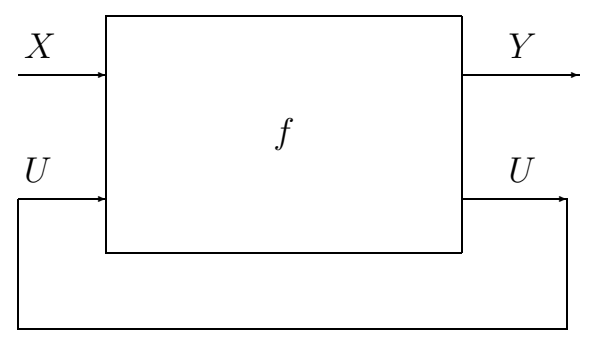

Examples relevant to this paper include Rel and Par, with $\otimes=\biguplus$, the disjoint union of sets. In the case of Par, the trace of a map $f: X \uplus U \rightarrow Y \uplus U$ is given by the following summation formula:

$$
\operatorname{Tr}_{X, Y}^{U}(f)=f_{X Y}+\sum_{n \in \mathbb{N}} f_{U Y} f_{U U}^{n} f_{X U}
$$

Here $f_{X Y}$ denotes the partial map $X \rightarrow Y$ obtained from $f$ by naturally restricting the domain and codomain (using injections and partial projections), and similarly for the other components. The sum of a family of partial maps $\sum_{n \in \mathbb{N}} h_{n}: X \rightarrow Y$ is defined iff the domains of the $h_{n}$ are disjoint, in which case $\left(\sum_{n \in \mathbb{N}} h_{n}\right)(x)=h_{k}(x)$ if $h_{k}(x) \downarrow$, and is undefined otherwise. Such traces given by the above formula are called "particle-style" ([1]) based on the following intuition: in the above picture imagine particles entering the box at $X$. Either they exit immediately at $Y$ via $f_{X Y}$ or they exit through $U$ and continue to cycle on $U$ some finite number $n$ times via $f_{U U}$ and then eventually exit at $Y$.

In [1], it is shown how a so-called GoI situation gives rise to a linear combinatory algebra. A GoI situation is a traced symmetric monoidal category equipped with a traced symmetric monoidal endofunctor, and an object $U$ satisfying various domain equations. By applying the GoI construction, one obtains a compact closed category containing an object whose points form a linear combinatory algebra. By the latter, one means an applicative structure $(A, \bullet)$ equipped with an endomap ! :A $\rightarrow A$ and several combinators, allowing for the application $(a, b) \mapsto a \bullet ! b$ to form a total combinatory algebra.

Lambek's register machines were described by a language of flowcharts and feedback. They can be naturally represented in a symmetric traced category with $\otimes=$ coproduct [58]. The original categorical studies of iterative notions of flowchart computation in a programming language setting was by C. Elgot. In this case iteration is given by a kind of feedback loop in a category whose hom-sets have infinite sums (Elgot's ideas are detailed in [91], and pursued more abstractly in traced $\Sigma$-monoid enriched tensor categories by Haghverdi [41]). Finally, traced monoidal categories in which the monoidal tensor $\otimes$ is obtained from a cartesian or genuine tensor product are discussed in [1], as well as a more general notion of partially traced categories, in [43].

7.2. Typed PCAs. The notions of computation considered so far has been untyped, in the sense that it is based on a single base type containing both the input/output values of computable maps and the (codes for) computable maps. In the notion of PCA, this is reflected by the fact that the partial 
application $a \bullet b$ regards $a$ as a code for a partial map and $b$ as an input. In various situations however, we do wish to consider computation over different types, for example because we wish to distinguish between the type of computable operations and the type of its inputs and outputs. One of the key concepts in the study of such situations is that of a typed PCA.

Definition 7.1 (Typed PCA). Let $T$ be the collection of simple types generated by a single base type N. A Typed Partial Combinatory Algebra (TPCA) over $T$ is a set-valued assignment $\tau \mapsto A(\tau)$ for $\tau \in T$, together with for all $\sigma, \tau \in T$ a partial application function $\bullet_{\sigma, \tau}: A(\sigma \rightarrow \tau) \times A(\sigma) \rightarrow A(\tau)$. As for PCAs, we write application using infix notation, associating to the left; we also suppress the typing information. One requires the existence of combinators

$$
\mathrm{k}_{\sigma, \tau} \in A(\sigma \rightarrow(\tau \rightarrow \sigma)) ; \quad \mathrm{s}_{\sigma, \tau, \rho} \in A((\sigma \rightarrow \tau \rightarrow \rho) \rightarrow((\sigma \rightarrow \tau) \rightarrow(\sigma \rightarrow \rho)))
$$

(for all types $\sigma, \tau, \rho)$ satisfying

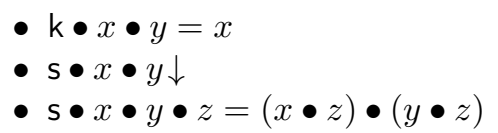

We remark that some authors also require the existence of fixed point combinators, numerals and recursors (see Lemma 5.10 for what these are in the untyped setting). This essentially guarantees that a TPCA is a model of Plotkin's simply typed programming language PCF (see [103]).

Example 7.2. Examples of TPCAs

(1) Let $A(N)=\mathbb{N}$, and $A(\sigma \rightarrow \tau)=A(\tau)^{A(\sigma)}$. Then we can let application be evaluation $\bullet$ : $A(\tau)^{A(\sigma)} \times A(\sigma) \rightarrow A(\tau)$. This is called the full (total) TPCA over $\mathbb{N}$.

(2) In the previous example we may instead let $A(\sigma \rightarrow \tau)=\operatorname{Par}(A(\sigma), A(\tau))$, the set of all partial functions. Then we get a TPCA where application is partial.

(3) If $\mathcal{C}$ is a CCC with NNO, we consider the subcategory on the simple types over the NNO. Taking global sections gives a TPCA.

(4) Any PCA $\mathcal{A}=(A, \bullet)$ is a typed PCA where $A(\sigma)=A$, and $\bullet \sigma, \tau=\bullet$ for all types $\sigma, \tau$.

(5) Term models of typed lambda calculus form TPCAs in the expected way, as do term models of programming languages based on typed lambda calculus, such as PCF.

Just as for PCAs, there is a notion of simulation between TPCAs. For example, to say that $\mathcal{A}$ has numerals (and that computable functions are representable) is to say that there is a simulation of Kleene's first model into $\mathcal{A}$. See [85] for details.

7.3. Computation at higher types. Most of the developments described above concern first-order computability (possibly taking place in a higher-order setting). We now briefly discuss computability at higher types. The relation between higher-order computability and first-order computability is analogous to that between functional analysis and analysis. Thus in higher-order computability one studies functionals $\mathbb{N}^{\mathbb{N}} \rightarrow \mathbb{N}$, and so on. Immediately, one recognizes the many possibilities: one could consider functionals acting on all total functions, or on all partial functions, or on all total computable functions, or on all partial computable functions, et cetera. We refer to the detailed survey paper [83] for a comprehensive historical overview.

Example 7.3. Kleene's S1-S9 One of the most fundamental notions of higher type computability was introduced in the landmark paper [62]. The collection of pure types over $\mathbb{N}$ is defined by:

$$
\mathbb{N}^{0}=1, \quad \mathbb{N}^{(k+1)}=\mathbb{N}^{\mathbb{N}^{(k)}} .
$$

Kleene's conditions S1-S9 define a class of partial maps of type

$$
\Phi: \mathbb{N}^{\left(k_{1}\right)} \times \cdots \times \mathbb{N}^{\left(k_{r}\right)} \rightarrow \mathbb{N} .
$$

More precisely, the definition specifies a relation $\{e\}\left(v_{1}, \ldots, v_{r}\right) \simeq x$, where $e \in \mathbb{N}$ is an index, the $v_{i}$ are elements of the pure types $\mathbb{N}^{\left(k_{i}\right)}$, and $x \in \mathbb{N}$. Thus the resulting definition is an example of partial functionals operating on total functions. 
Another classic example of a notion of computation at higher type, first introduced in [63], is the following:

Example 7.4. Hereditarily Effective Operations Define simultaneously, for each simple type over the natural numbers, a set of natural numbers and an equivalence relation on the set as follows:

- $\mathbf{H E O}_{0}=\mathbb{N}$, and $n \sim_{0} m \Leftrightarrow n=m$.

- $\mathbf{H E O}_{\sigma \rightarrow \tau}=\left\{e \in \mathbb{N} \mid \phi_{e}\right.$ induces a total function $\left.\mathbf{H E O} \mathbf{O}_{\sigma} \rightarrow \mathbf{H E O}_{\tau}\right\}$, and $e \sim_{\sigma \rightarrow \tau} e^{\prime} \Leftrightarrow \forall n \in$ $\mathbf{H E O}_{\sigma} \cdot \phi_{e}(n) \sim_{\tau} \phi_{e^{\prime}}(n)$.

One of the central contributions in [85] is the development of a general framework (called computability model) for studying the wide variety of possible notions of higher type computation. It also supports a general notion of simulation between models, and of equivalence. Typed PCAs form an important class of examples of computability models. We shall now sketch a result by Longley characterizing the so-called extensional collapse of a large family of TPCAs. From now on, we assume our TPCAs come equipped with a choice of numerals $\mathbb{N} \rightarrow A(N)$.

Definition 7.5 (Extensional Collapse of a TPCA). Let $\mathcal{A}$ be a TPCA. Define, at each simple type $\sigma$, a PER $\sim_{\sigma}$ on $A(\sigma)$ as follows:

- $a \sim_{N} b$ iff $a=b=\bar{n}$ for some $n \in \mathbb{N}$

- $a \sim_{\sigma \rightarrow \tau} b$ iff for all $x, y \in A(\sigma)$ with $x \sim_{\sigma} y, a \bullet x \sim_{\tau} b \bullet y$.

The sets $A(\sigma) / \sim_{\sigma}$ form a simple type structure over $\mathbb{N}$, denoted $\mathbf{E C}(\mathcal{A})$.

Definition 7.6. A typed PCA $\mathcal{A}$ is

(i) continuous if there is a numeral-respecting simulation $\mathcal{A} \rightarrow \mathcal{K}_{2}$;

(ii) full continuous if it is continuous and all functions $\mathbb{N} \rightarrow \mathbb{N}$ are represented in $\mathcal{A}$;

(iii) effective if there is a numeral-respecting simulation $\mathcal{A} \rightarrow \mathcal{K}_{1}$;

The following general result (referred to as the Ubiquity Theorem) now describes the extensional collapse of these important classes of typed PCAs:

Theorem 7.7 (Longley [84]). Let $\mathcal{A}$ be a typed PCA.

(i) If $\mathcal{A}$ is full continuous, then $\mathbf{E C}(\mathcal{A})=\mathrm{C}$, the total continuous functionals (which may be taken to be $\left.\mathbf{C}=\mathbf{E C}\left(\mathcal{K}_{2}\right)\right)$.

(ii) If $\mathcal{A}$ is effective (and satisfies a few minor technical conditions), then $\mathbf{E C}(\mathcal{A})=\mathbf{H E O}$, the hereditarily effective operations.

There is a third part to the theorem, which characterizes the collapse of a class of relative TPCAs. By the latter, we mean a TPCA $\mathcal{A}$ together with a sub-TPCA $\mathcal{A}^{\#}$, that is, a collection of subsets $A^{\#}(\sigma) \subseteq$ $A(\sigma)$ closed under the application and containing the combinators $\mathbf{k}, \mathbf{s}$. There is a corresponding relative version of the extensional collapse. Longley's third theorem then states that when $\left(\mathcal{A}, \mathcal{A}^{\#}\right)$ is a relative TPCA with $\mathcal{A}$ full continuous and $\mathcal{A}^{\#}$ effective, $\operatorname{EC}\left(\mathcal{A}, \mathcal{A}^{\#}\right)=\mathrm{RC}$, the total recursive continuous functionals. The latter may be taken to be $\mathbf{E C}\left(\mathcal{K}_{2}, \mathcal{K}_{2}^{\mathrm{eff}}\right)$.

7.4. Higher-order computation in toposes. Since toposes are cartesian closed we can also consider higher type computability in toposes. Let us consider this first in the case of the effective topos. The following result already appears in [52]:

Theorem 7.8 (Hyland [52]). The total functionals of higher type over the NNO in Eff are precisely the hereditarily effective operations.

Next, consider the Mulry topos; this is the topos of sheaves on the monoid of total computable functions, with the canonical topology. (The latter amounts to taking as basic coverings sets $\left\{f_{1}, \ldots, f_{k}\right\}$ for which $\bigcup_{i=1}^{k} \operatorname{Im}\left(f_{i}\right)=\mathbb{N}$.) For the following result, a functional $G: \mathbb{N}^{\mathbb{N}} \rightarrow \mathbb{N}$ is called Banach-Mazur when for each computable $h: \mathbb{N}^{2} \rightarrow \mathbb{N}$, the composite $G \circ \tilde{h}$ is computable, where $\tilde{h}: \mathbb{N} \rightarrow \mathbb{N}^{\mathbb{N}}$ is the transpose of $h$. 
Theorem 7.9 (Mulry [96]). The functionals $\mathrm{N}^{\mathrm{N}} \rightarrow \mathrm{N}$ in the Mulry topos are precisely the Banach-Mazur functionals.

Finally, let us consider the free topos. What are the total functionals of pure type in the free topos, i.e. arrows $\mathrm{N}^{(k)} \rightarrow \mathrm{N}^{(\ell)}, k, \ell \geq 1$ ? This question is answered in an interesting paper of A. Scedrov [109].

Theorem 7.10 (Scedrov [109]). Let $\mathcal{F}$ be the free topos, let $\mathcal{C}$ be the free $C C C$ with $N N O$ and let $\mathcal{F}_{\mathcal{C}}$ be the full subcategory of $\mathcal{F}$ generated by $\mathcal{C}$. The morphisms of $\mathcal{F}_{\mathcal{C}}$ are precisely those Kleene computable functionals that are provably total in the internal logic of $\mathcal{F}$.

The proof uses a gluing (or Friedman Realizability) argument (cf. [76, 77, 118]) together with induction. The ambient set theory is the free topos $\mathcal{F}$ itself, and, for each type level $j \geq 0$ we construct an Effective topos $\mathbf{E f f}(j)$ internally in the free topos, gluing it along a certain left exact functor $\Delta: \operatorname{Eff}(j) \rightarrow \mathcal{F}$.

7.5. Complexity Theory. While classical computability theory is often concerned with the degree of unsolvability of various problems, the branch most relevant to computer science is that of complexity theory, where one classifies solvable problems according to the time and/or resources their solutions require. In particular, one is interested in complexity classes and the connections between those. For example, the class PTIME consists of problems whose solution (regarded as a function of the input value $n \in \mathbb{N}$ ) requires $p(n)$ steps (of a deterministic Turing machine, say), where $p$ is a polynomial with positive integer coefficients. We refer to [44] for an introduction.

Early work in Implicit Computational Complexity by Martin Hofmann e.g. [48] used complexitybounded combinatory algebras and realizability to study logics of bounded complexity. A BCK algebra is an applicative structure $\mathcal{A}$ having the combinators b, c, k, where (still associating to the left)

$$
\mathrm{b} x y z=x(y z) ; \quad \text { c } x y z=x z y ; \quad \mathrm{k} x y=x .
$$

Any total PCA is a BCK algebra, but not vice versa: the diagonal $x \mapsto x x$ is generally not computable in a BCK algebra. One of the results in [48] shows that there is a BCK algebra structure on the natural numbers capturing PTIME computation:

Theorem 7.11 (Hofmann [48]). There exists a BCK algebra structure on $\mathbb{N}$ such that the computable maps w.r.t. this structure are precisely the polynomial-time computable functions.

Related applications of such bounded combinatory algebras (to reprove the theorem that the representable functions of Bounded Linear Logic are exactly those in PTIME) appear in [49].

Recent work in Turing categories has focussed on the following general question: which complexity classes (e.g. LINEAR, PTIME, LOGSPACE, etc.) can occur as the total maps of a Turing Category? Of course, such a Turing category cannot be a subcategory of Par, since it would then necessarily contain all total computable functions. Hence, it has to be a category whose global sections functor is not faithful.

The paper [24] explores the area in more detail. Their main theorem characterizes when a Cartesian Category $\mathcal{C}$ with a Universal Object $U$, a pair of disjoint elements $\{t, f\}$, and various abstract coding retract structure can arise as the total maps of a Turing Category. The construction makes use of the idea that the given retract structure allows one to simulate a simple programming language. Passing to the presheaf topos of $\mathcal{C}$ then provides the required structure of a trace (on the coproduct) for implementing this language to obtain a PCA.

As a consequence of this characterization, one obtains the following corollary:

Corollary 7.12. Any countable Cartesian category with a universal object $U$ and a pair of disjoint elements is the total maps of a Turing category.

In order to apply this result to show that a particular complexity class arises as the total maps of a Turing category, one is thus required to establish that the class in question admits the required closure 
conditions and pairing operations. For example, the classes of LINEAR and PTIME maps (between binary numbers) can be shown to meet these requirements [24]. However, it is not fully understood for which complexity classes this is possible.

\section{CONCLUSION}

We hope that we have shown in this -admittedly biased- overview of categorical recursion theory how various of Lambek's seminal ideas have initiated and inspired numerous strands of research that are still being pursued today.

We also hope to have conveyed to the reader that there are still many interesting unanswered questions and relatively unexplored facets of categorical recursion theory that deserve further investigation.

\section{REFERENCES}

[1] S. Abramsky, E. Haghverdi, and P. Scott. Geometry of interaction and linear combinatory algebras. Math. Structures in Computer Science, 12:1-40, 2002.

[2] R. M. Amadio and P.-L. Curien. Domains and lambda-calculi. Cambridge University Press, 1998.

[3] W. S. Anglin and J. Lambek. The Heritage of Thales. Undergraduate Texts in Mathematics. Springer, 1995.

[4] M. Artin, A. Grothendieck, and J.-L. Verdier, editors. SGA4: Théorie des topos et cohomologie étale des schémas. Number 269, 270, 305 in Lecture Notes in Mathematics. Springer, 1972/3.

[5] S. Awodey. Category Theory. Number 52 in Oxford Logic Guides. Oxford University Press, 2 edition, 2010.

[6] H. P. Barendregt. The lambda calculus: its syntax and semantics, volume 103 of Studies in Logic and the Foundations of Mathematics. Elsevier, Amsterdam, 1984. Revised edition.

[7] I. Bethke. Notes on Partial Combinatory Algebras. PhD thesis, Universiteit van Amsterdam, 1988.

[8] M. Bhargava and J. Lambek. A rewrite system of the Western Pacific: Lounsbury's analysis of trobriand kinship terminology. Theoretical Linguistics, 21(2-3):241-253, 1995.

[9] R. Blute, J. R. B. Cockett, and R. A. G. Seely. Categories for computation in context and unified logic. J. Pure and Applied Algebra, 116:49-98, 1997.

[10] R. Blute, J. R. B. Cockett, R. A. G. Seely, and T. Trimble. Natural deduction and coherence for weakly distributive categories. J. Pure and Applied Algebra, 3(113):229-296, 2002.

[11] G. Boolos, J. Burgess, and R. Jeffrey. Computability and Logic. Cambridge University Press, 4th edition, 2007.

[12] A. Burroni. Récursivité graphique (1ère partie): catégorie des fonctions récursives primitives formelles. Cah. Topol. Géom. Différ. Catég, 27(1):49-79, 1986.

[13] A. Carboni. Some free constructions in realizability and proof theory. Journal of Pure and Applied Algebra, 103:117148, 1995.

[14] A. Carboni, P. J. Freyd, and A. Scedrov. A categorical approach to realizability and polymorphic types. Lecture Notes in Computer Science, 298:23-42, 1988.

[15] A. Carboni, J. Lambek, and M. C. Pedicchio. Diagram chasing in Mal'cev categories. Journal of Pure and Applied Algebra, 69(3):271-284, 1990.

[16] A. Carboni and E. M. Vitale. Regular and exact completions. Journal of Pure and Applied Algebra, 125(1-3):79-116, 1998.

[17] S. Castellan, P. Clairambault, and P. Dybjer. Categories with families: unityped, simply typed, and dependently typed. 2019.

[18] J. R. B. Cockett. Categories and Computability: Notes for the Estonia Winter School. http://pages.cpsc.ucalgary.ca/ robin/, 2010.

[19] J. R. B. Cockett, X. Guo, and P. J. W. Hofstra. Range categories I: General theory. Theory and Applications of Categories, 26:412-452, 2012.

[20] J. R. B. Cockett, X. Guo, and P. J. W. Hofstra. Range categories II: Towards regularity. Theory and Applications of Categories, 26:453-500, 2012.

[21] J. R. B. Cockett and P. J. W. Hofstra. Introduction to Turing categories. Annals of Pure and Applied Logic, 2007.

[22] J. R. B Cockett and P. J. W. Hofstra. Categorical simulations. Journal of Pure and Applied Algebra, 214(10):1835$1853,2010$.

[23] J. R. B. Cockett and P. J. W. Hofstra. Unitary theories, unitary categories. Electronic Notes in Theoretical Computer Science, 2010.

[24] J. R. B. Cockett, P. J. W. Hofstra, and P. Hrubes. Total maps of Turing categories. ENTCS, 308:129-146, 2014.

[25] J. R. B Cockett and S. Lack. Restriction categories I. Theoretical Computer Science, 270:223-259, 2002.

[26] J. R. B. Cockett and R. A. G. Seely. Proof theory for full intuitionistic linear logic, bilinear logic and mix categories. Theory and Applications of Categories, 3(5):85-131, 1997.

[27] N. J. Cutland. Computability. Cambridge University Press, 1980.

[28] R. Dedekind. Was sind und sollen die Zahlen? Braunschweig: Vieweg, 1888.

[29] K. Došen. Cut Elimination in Categories, volume 6 of Trends in Logic. Kluwer, Dordregt, 1999. 
[30] K. Došen and Z. Petric. Proof-Theoretical Coherence, volume 1 of Studies in Logic. King's College Publications, 2004.

[31] K. Došen and Z. Petric. Proof-Net Categories. Polimetrica, Monza, 2007.

[32] S. Eilenberg and C. C. Elgot. Recursiveness. Academic Press, 1970.

[33] S. Feferman. A language and axioms for explicit mathematics. In J. N. Crossley, editor, Algebra and Logic, pages 87-139. Springer-Verlag, 1975.

[34] M. P. Fourman and D. S. Scott. Sheaves and logic. In C.J. Mulvey M.P. Fourman and D.S. Scott, editors, Applications of Sheaves, volume 753 of Lecture Notes in Mathematics, pages 302-401. Springer-Verlag, 1979.

[35] J. Frey. Characterizing partitioned assemblies and realizability toposes. Journal of Pure and Applied Algebra, 223(5):2000-2014, 2019.

[36] J.-Y. Girard. Linear logic. Theoretical Computer Science, 50:1-102, 1987.

[37] J.-Y. Girard, Y. Lafont, and P. Taylor. Proofs and Types. Cambridge University Press, 1989.

[38] K. Gödel. Über formal unentscheidbare Sätze der Principia Mathematica und verwandter Systeme, I. Monadshefte für Mathematik und Physik, 38(1), 173-198 1931.

[39] K. Gödel. Über eine bisher noch nicht benützte Erweiterung des finiten Standpunktes. Dialectica, 12(3/4):280-287, 1958.

[40] R. L. Goodstein. Recursive Number Theory. Studies in Logic and the Foundations of Mathematics. Elsevier, Amsterdam, 1957.

[41] E. Haghverdi. A Categorical Approach to Linear Logic, Geometry of Proofs and Full Completeness. PhD thesis, University of Ottawa, 2000.

[42] E. Haghverdi and P. Scott. A categorical model for the geometry of interaction. Theoretical Computer Science, 350:252-274, 2006.

[43] E. Haghverdi and P. J. Scott. Towards a typed geometry of interaction. Math. Structures in Comp. Science, 20(3):149, 2010.

[44] D. Harel and Y. Feldman. Algorithmics. Addison-Welsey, 3rd edition, 2004.

[45] A. Heller. An existence theorem for recursion categories. Journal of Symbolic Logic, 55(3):1252-1268, 1990.

[46] D. Higgs. A category approach to boolean-valued set theory. Technical report, University of Waterloo, 1973.

[47] D. Hilbert and P. Bernays. Grundlagen der Matematik I. Number 40 in Die Grundlehren der mathematische Wissenschaften. Springer-Verlag, 1934.

[48] M. Hofmann. Safe recursion with higher types and BCK-algebra. Annals of Pure and Applied Logic, 104(3):113-166, 2000.

[49] M. Hofmann and P. J. Scott. Realizability models for BLL-like languages. Theoretical Computer Science, 318:121137,2004

[50] P. J. W. Hofstra and J. van Oosten. Ordered partial combinatory algebras. Mathematical Proceedings of the Cambridge Philosophical Society, 134:445-463, 2003.

[51] V. Huber-Dyson. Strong representability of number-theoretic functions. Technical report, Hughes Aircraft, 1965.

[52] J. M. E. Hyland. The effective topos. In A.S. Troelstra and D. Van Dalen, editors, The L.E.J. Brouwer Centenary Symposium, pages 165-216. North Holland Publishing Company, 1982.

[53] P. T. Johnstone J. M. E. Hyland and A. M. Pitts. Tripos theory. Mathematical Proceedings of the Cambridge Philosophical Society, 88:205-232, 1980.

[54] C. B. Jay. Languages for monoidal categories. Journal of Pure and Applied Algebra, 59:61-85, 1989.

[55] C. B. Jay. The structure of free closed categories. Journal of Pure and Applied Algebra, 66:271-285, 1990.

[56] A. Joyal. The Gödel incompleteness theorem, a categorical approach (abstract). Cah. de Top. Geom. Diff., 16(3), 2005.

[57] A. Joyal, R. Street, and D. Verity. Traced monoidal categories. Mathematical Proceedings of the Cambridge Philosophical Society, 119:447-468, 1996.

[58] P. Katis, N. Sabadini, and R. F. C. Walters. Feedback, trace, and fixed-point semantics. Theoretical Informatics and Applications, 36(2):181-194, 2002.

[59] G. M. Kelly and S. Mac Lane. Coherence in closed categories. J. Pure and Applied Algebra, 1(1):97-140, 1971.

[60] S. C. Kleene. On the interpretation of intuitionistic number theory. Journal of Symbolic Logic, 53(1):109-124, 1945.

[61] S. C. Kleene. Introduction to Metamathematics. North Holland, 1952.

[62] S. C. Kleene. Recursive functionals and quantifiers of finite types I. Transactions of the American Mathematical Society, 91(1):1-52, 1959.

[63] G. Kreisel. Interpretation of analysis by means of constructive functionals of finite types. In A. Heyting, editor, Constructivity in mathematics: proceedings of the colloquium held in Amsterdam, pages 101-128. North-Holland, Amsterdam, 1959.

[64] J. Lambek. The mathematics of sentence structure. Amer. Math. Monthly, 65:154-169, 1958.

[65] J. Lambek. How to program an infinite abacus. Canadian Mathematical Bulletin, 4(3):295-302, 1961.

[66] J. Lambek. On the calculus of syntactic types, volume 12 of Proc. Symposium Appl. Math., pages 166-178. AMS, 1961.

[67] J. Lambek. Deductive systems and categories I. J. Math. Syst. Theory, 1968.

[68] J. Lambek. A fixpoint theorem for complete categories. Math. Zeitschrift, 103:151-161, 1968. 
[69] J. Lambek. Deductive Systems and Categories II, volume 86 of Lecture Notes in Mathematics, pages 76-122. Springer, 1969.

[70] J. Lambek. Deductive Systems and Categories III, volume 274 of Lecture Notes in Mathematics, pages 57-82. Springer, 1972.

[71] J. Lambek. Functional completeness of cartesian categories. Annals of Mathematical Logic, 6(3):259 - 292, 1974.

[72] J. Lambek. From $\lambda$-calculus to cartesian closed categories. In J. P. Seldin and J. R. Hindley, editors, To $H$. $B$. Curry, Essays on Combinatory Logic, Lambda Calculus and Formalism, pages 375-402. Academic Press, 1980.

[73] J. Lambek. Multicategories revisited. Contemp. Mathematics, 92:217-239, 1989.

[74] J. Lambek. Relations in operational categories. J. Pure and Applied Algebra, 116:221-248, 1997.

[75] J. Lambek and P. J. Scott. An exactification of the monoid of primitive recursive functions. Studia Logica, 81(1):1-18, 2005.

[76] J. Lambek and P.J. Scott. Intuitionist type theory and the free topos. Journal of Pure and Applied Algebra, 19:215$257,1980$.

[77] J. Lambek and P.J. Scott. Introduction to higher order categorical logic, volume 7 of Cambridge studies in advanced mathematics. Cambridge University Press, 1986.

[78] F. W. Lawvere. An elementary theory of the category of sets. (extended version published in TAC reprints: http://www.tac.mta.ca/tac/). Proceedings of the National Academy of Science of the U.S.A, 52:1506-1511, 1964.

[79] F. W. Lawvere. Diagonal arguments and cartesian closed categories. In Category Theory, Homology Theory and their Applications, II (Battelle Institute Conference, Seattle, Wash., 1968, Vol. Two), pages 134-145. Springer-Verlag, Berlin, 1969.

[80] F. W. Lawvere. Quantifiers and sheaves. In Actes du ICM, Nice 1970, I, pages 329-334. Gauthier-Villars, Paris, 1971.

[81] S. Lee and J. van Oosten. Basic subtoposes of the effective topos. Annals of Pure and Applied Logic, 164(9):866-883, 2013.

[82] J. Longley. Realizability toposes and language semantics. PhD thesis, University of Edinburgh, 1994.

[83] J. Longley. Notions of computability at higher types I. In R. Cori, A. Razborov, S. Todorčević, and C. Wood, editors, Logic Colloquium 2000, volume 19 of Lecture Notes in Logic, pages 32-142. Cambridge University Press, 2000.

[84] J. Longley. On the ubiquity of certain total type structures. Mathematical Structures in Computer Science, 17(5):841-953, 2007.

[85] J. Longley and D. Normann. Higher-Order Computability. Springer, 2015.

[86] G. Longo and E. Moggi. A category theoretic characterization of functional completeness. Theoretical Computer Science, 70(2):193-211, 1990.

[87] S. Mac Lane. Why commutative diagrams coincide with equivalent proofs. Contemp. Mathematics, 13:387-401, 1982.

[88] S. Mac Lane. Categories for the Working Mathematician. Graduate Texts in Mathematics. Springer, 1998.

[89] M. Maietti. Joyal's arithmetic universe as list-arithmetic pretopos. Theory and Applications of Categories, 24(3):3983, 2010.

[90] M. Maietti and S. Vickers. An induction principle for consequence in arithmetic universes. Journal of Pure and Applied Algebra, 216:2049-2067, 2012.

[91] E. Manes and M. Arbib. Algebraic Approaches to Program Semantics. Springer-Verlag, 1986.

[92] M. Menni. A characterization of the left exact categories whose exact completions are toposes. Journal of Pure and Applied Algebra, 177(3):287-301, 2003.

[93] G. E. Mints. Closed categories and the theory of proofs. Zap. Naućn Seminar Leningrad Otdel Mat. Inst. Steklov (LOMI), 68:83-114, 1977.

[94] G. E. Mints. Proof theory and category theory (in russian). Aktual'nye voprosy logiki i metodologii nauki, Naukova Dumka, Kiev, pages 252-278, 1980.

[95] G. E. Mints. Selected Papers in Proof Theory. Number 3 in Studies in Proof Theory. North-Holland, 1992.

[96] P. S. Mulry. Generalized Banach-Mazur functionals in the topos of recursive sets. Journal of Pure and Applied Algebra, 26(71-83), 1982.

[97] P. Odifreddi. Classical recursion theory, volume 125 of Studies in Logic. North-Holland, 1989.

[98] R. Di Paola and A. Heller. Dominical categories: recursion theory without elements. Journal of Symbolic Logic, 52:595-635, 1987.

[99] R. Di Paola and F. Montagna. Some properties of the syntactic p-recursion categories generated by consistent, recursively enumerable extensions of Peano arithmetic. Journal of Symbolic Logic, 56(2):643-660, 1991.

[100] R. Paré and L. Román. Monoidal categories with natural numbers object. Studia Logica, 48(3), 1989.

[101] R. Péter. Recursive Functions. Academic Press, 1967.

[102] W. Phoa. Relative computability in the effective topos. Mathematical Proceedings of the Cambridge Philosophical Society, 106:419-422, 1989.

[103] G. D. Plotkin. LCF considered as a programming language. Theoretical Computer Science, 5:223-255, 1977. 
[104] G. D. Plotkin. Partial recursive functions and finality. In L. Ong B. Coecke and P. Panangaden, editors, Computation, Logic, Games, and Quantum Foundations. The Many Facets of Samson Abramsky, volume 7860 of Lecture Notes in Computer Science, pages 311-326. Springer, 2013.

[105] E. Post. Recursively enumerable sets of positive integers and their decision problems. Bulletin of the American Mathematical Society, 50:284-213, 1944.

[106] E. P. Robinson and G. Rosolini. Colimit completions and the effective topos. Journal of Symbolic Logic, 55(2):678699, 1990.

[107] E. P. Robinson and G. Rosolini. An abstract look at realizability. In Laurent Fribourg, editor, Computer Science Logic, 15th International Workshop (CSL 2001), volume 2142 of Lecture Notes in Computer Science, pages $173-187$. Springer, 2001.

[108] L. Román. Cartesian categories with natural numbers object. Journal of Pure and Applied Algebra, 58:267-278, 1989.

[109] A. Scedrov. Kleene computable functionals and the higher order existence property. Journal of Pure and Applied Algebra, 52:313-320, 1988.

[110] D. Scott. Relating theories of the lambda calculus. In J. P. Seldin and J. R. Hindley, editors, To H. B. Curry, Essays on Combinatory Logic, Lambda Calculus and Formalism, pages 403-450. Academic Press, 1980.

[111] T. Skolem. The foundations of elementary arithmetic. In J. van Heijenoort, editor, From Frege to Gödel: A Source Book in Mathematical Logic, 1879-1931, pages 302-333. Harvard University Press, 1967 (1923).

[112] R. Soare. Computability and recursion. Bulletin of Symbolic Logic, 2(3):284-321, 1996.

[113] A. S. Troelstra, editor. Metamathematical Investigation of Intuitionistic Arithmetic and Analysis, volume 344 of LNCS. Springer Verlag, 1973.

[114] A. S. Troelstra and H. Schwichtenberg. Basic Proof Theory. Cambridge Tracts in Theoretical Computer Science. Cambridge University Press, 2 edition, 2000.

[115] J. van Oosten. Realizability: a historical essay. Mathematical Structures in Computer Science, 12:239-263, 2002.

[116] J. van Oosten. Realizability: an introduction to its categorical side, volume 152 of Studies in Logic. North-Holland, 2008.

[117] P. Vinogradova. Investigating computability in Turing categories. Master's thesis, University of Ottawa, 2011.

[118] G. C. Wraith. Artin gluing. Journal of Pure and Applied Algebra, 4:345-348, 1974.

[119] N. Yanofsky. A universal approach to self-referential paradoxes, incompleteness and fixed points. Bulletin of Symbolic Logic, 09(3):362-386, 2003.

Department of Mathematics and Statistics, University of Ottawa, Canada,

E-mail address: phofstra@uottawa.ca

E-mail address: phil@site.uottawa.ca 